\title{
Quasidense monotone multifunctions
}

\author{
Stephen Simons *
}

\begin{abstract}
In this paper, we discuss quasidense multifunctions from a Banach space into its dual, and use the two sum theorems proved in a previous paper to give various characterizations of quasidensity. We investigate the Fitzpatrick extension of such a multifunction. We prove that, for closed monotone multifunctions, quasidensity implies type (FPV) and strong maximality, and that quasidensity is equivalent to type (FP).
\end{abstract}

2010 Mathematics Subject Classification: Primary 47H05; Secondary 47N10, 52A41, 46A20.

Keywords: Multifunction, maximal monotonicity, quasidensity, sum theorem, subdifferential, strong maximality, type (FPV), type (FP).

\section{Introduction}

This is a sequel to the paper 23, in which we introduced the concepts of Banach $S N$ space, L-positive set, $r_{L}$-density and Fitzpatrick extension. In this paper we suppose that $E$ is a nonzero real Banach space with dual $E^{*}$, and we apply some of the results of 23 to the Banach SN space $E \times E^{*}$. In this case, $L-$ positive means the same as monotone and we use the word quasidense to stand for $r_{L}$-dense.

In Section 2, we introduce some general Banach space notation, and give the concepts and results from 23 that we shall use. Let $S: E \rightrightarrows E^{*}$ be a multifunction. We define the quasidensity of $S$ in Definition 2.1. We point out in Theorem 2.2 and Example 2.3 that every closed, monotone quasidense multifunction is maximally monotone, but that there exists maximally monotone linear operators that are not quasidense. We define the function $\varphi_{S}$ in Definition 2.4. ( $\varphi_{S}$ is the "Fitzpatrick function" of $S$, which was originally introduced in the Banach space setting in [4. (1988)], but lay dormant until it was rediscovered by Martínez-Legaz and Théra in [12, (2001)]. It had been previously considered in the finite-dimensional setting by Krylov in 9.) In Theorem 2.7. we give a criterion in terms of the Fenchel conjugate, $\varphi_{S}{ }^{*}$, of $\varphi_{S}$ for a maximally monotone multifunction to be quasidense (other criteria can be

*Department of Mathematics, University of California, Santa Barbara, CA 93106-3080, U.S.A. Email: stesim38@gmail.com. 
found in Theorems 6.1, 6.2 and 8.3). In Theorem 2.14 we prove that the subdifferential of a proper, convex, lower semicontinuous function on $E$ is quasidense (thus generalizing Rockafellar's result, [16, (1970)]). The rest of Section 2 is devoted to the discussion of two significant examples that will be used in later parts of the paper.

If $S: E \rightrightarrows E^{*}$ is closed, monotone and quasidense then, in Section [3, we define an associated maximally monotone multifunction $S^{\mathbb{F}}: E^{*} \rightrightarrows E^{* *}$, which we call the Fitzpatrick extension of $S . S^{\mathbb{F}}$ is defined formally in terms of $\varphi_{S}{ }^{*}$ in Definition 3.1, and we give three characterization of $S^{\mathbb{F}}$ in Theorem 7.5 and two in (9.3) and (9.4). (It is observed in Appendix 1, Section 9, that $\left(y^{*}, y^{* *}\right) \in G\left(S^{\mathbb{F}}\right)$ exactly when $\left(y^{* *}, y^{*}\right)$ is in the Gossez extension of $\left.G(S)\right)$. Now let $f$ be a proper, convex, lower semicontinuous function on $E$. We prove in Lemma 3.3 that $G\left((\partial f)^{\mathbb{F}}\right) \subset G\left(\partial\left(f^{*}\right)\right)$. Lemma 3.3 will be used several times in subsequent parts of the paper. In Theorem 3.7, we strengthen Lemma 3.3 and prove that, in fact, $(\partial f)^{\mathbb{F}}=\partial\left(f^{*}\right)$. Theorem 3.7 will be used in Theorems 3.8 and 3.9 to characterize the Fitzpatrick extensions of the two examples introduced in Section 2.

In Section 4. we state the Sum theorem with domain constraints and the Sum theorem with range constraints that were established in [23].

In Section 5, we prove that every closed, monotone quasidense multifunction is of type $(F P V)$. (Type (FPV) was introduced independently by FitzpatrickPhelps and Verona-Verona in [6, p. 65(1995)] and [26, p. 268(1993)] under the name of "maximal monotone locally".)

In Section 6, we give two "fuzzy" criteria for quasidensity in which an element of $E^{*}$ is replaced by a nonempty $w\left(E^{*}, E\right)$-compact convex subset of $E^{*}$, or an element of $E$ is replaced by a nonempty $w\left(E, E^{*}\right)$-compact convex subset of $E$. We refer the reader to the introduction to Section 6 for more precise details of these results. These two fuzzy criteria are applied in Theorem 6.5 to prove that every closed, monotone quasidense multifunction is strongly maximal in the sense of [17. Theorems 6.1-2, pp. 1386-1387].

In Section [7, we give sequential characterizations of $S^{\mathbb{F}}$ (see Theorem 7.5 ). In view of Appendix 1, Section 9. Theorem 7.5 actually provides sequential characterizations of the Gossez extension of $S$.

In Section 8 , we prove that a maximally monotone multifunction is quasidense if, and only if, it is of type (FP). (Type (FP) was introduced by FitzpatrickPhelps in [5, Section 3(1992)] under the name of "locally maximal monotone".)

In Appendix 2, Section [10, we indicate how the results of Section 8 can be obtained without appealing to the results of Section 4 , but using instead two results of Rockafellar.

There are many classes of maximally monotone multifunctions that we will not discuss in great detail in this paper, they share the common feature that they all require the bidual, $E^{* *}$, of $E$ for their definition: Type $(D)$ and dense type were introduced by Gossez (see Gossez, 8, Lemme 2.1, p. 375(1971)] and Phelps, 14. Section 3(1997)] for an exposition). Type (NI) was first defined in [18. Definition 10, p. 183(1996)], and type (ED) was introduced in [19, (1998)], 
(under the name of type $(D S)$ ). Because of the work of Voisei and Zălinescu, [27, Marques Alves and Svaiter, [10. Theorem 4.4, pp. 1084-1085(2010)], Simons, [21, Theorem 9.9(a), pp. 254-255(2011)] and Bauschke, Borwein, Wang and Yao, 2, Theorem 3.1, pp. 1878-1879(2012)] we now know that type (D), dense type, type (FP), type (NI) and type (ED) are all equivalent. In this paper, we will only discuss in detail classes of the maximally monotone multifunctions, like quasidensity, that can be defined without reference to the bidual.

The bidual is not mentioned explicitly in the statements of Theorems 4.1 5.2 6.1 6.2, 6.5 and 8.3( (a) $\Longleftrightarrow(\mathrm{b}))$, but our proofs of all of these results ultimately depend on the bidual at one point or another. This raises the fascinating question whether there are proofs of any of these results that do not depend on the bidual. This seems quite a challenge, because it would require finding substitutes for Theorems 4.1 and 4.2

The author would like to thank Mircea Voisei and Regina Burachik for some very helpful comments on an earlier version of this paper.

\section{Basic notation and definitions}

If $X$ is a nonzero real Banach space and $f: X \rightarrow]-\infty, \infty]$, we write $\operatorname{dom} f$ for the set $\{x \in X: f(x) \in \mathbb{R}\}$. $\operatorname{dom} f$ is the effective domain of $f$. We say that $f$ is proper if $\operatorname{dom} f \neq \emptyset$. We write $\mathcal{P C} \mathcal{L S C}(X)$ for the set of all proper convex lower semicontinuous functions from $X$ into $]-\infty, \infty]$. We write $X^{*}$ for the dual space of $X$ (with the pairing $\langle\cdot, \cdot\rangle: X \times X^{*} \rightarrow \mathbb{R}$ ). If $f \in \mathcal{P C} \mathcal{L} \mathcal{S C}(X)$ then, as usual, we define the Fenchel conjugate, $f^{*}$, of $f$ to be the function on $X^{*}$ given by $x^{*} \mapsto \sup _{X}\left[x^{*}-f\right]$. We write $X^{* *}$ for the bidual of $X$ (with the pairing $\left.\langle\cdot, \cdot\rangle: X^{*} \times X^{* *} \rightarrow \mathbb{R}\right)$. If $f \in \mathcal{P C} \mathcal{L S C}(X)$ and $f^{*} \in \mathcal{P C} \mathcal{L S C}\left(X^{*}\right)$, we define $\left.\left.f^{* *}: X^{* *} \rightarrow\right]-\infty, \infty\right]$ by $f^{* *}\left(x^{* *}\right):=\sup _{X^{*}}\left[x^{* *}-f^{*}\right]$. If $x \in X$, we write $\widehat{x}$ for the canonical image of $x$ in $X^{* *}$, that is to say, for all $\left(x, x^{*}\right) \in X \times X^{*}$, $\left\langle x^{*}, \widehat{x}\right\rangle=\left\langle x, x^{*}\right\rangle$. If $f \in \mathcal{P C} \mathcal{L S C}(X)$ then the subdifferential of $f$ is the multifunction $\partial f: E \rightrightarrows E^{*}$ that satisfies $x^{*} \in \partial f(x) \Longleftrightarrow f(x)+f^{*}\left(x^{*}\right)=\left\langle x, x^{*}\right\rangle$. We write $X_{1}$ for the closed unit ball of $X$.

We now collect the definitions and results from [23] that we shall use. We suppose that $E$ is a nonzero real Banach space with dual $E^{*}$. For all $\left(x, x^{*}\right) \in$ $E \times E^{*}$, we write $\left\|\left(x, x^{*}\right)\right\|:=\sqrt{\|x\|^{2}+\left\|x^{*}\right\|^{2}}$. We represent $\left(E \times E^{*}\right)^{*}$ by $E^{*} \times E^{* *}$, under the pairing

$$
\left\langle\left(x, x^{*}\right),\left(y^{*}, y^{* *}\right)\right\rangle:=\left\langle x, y^{*}\right\rangle+\left\langle x^{*}, y^{* *}\right\rangle .
$$

The dual norm on $E^{*} \times E^{* *}$ is given by $\left\|\left(y^{*}, y^{* *}\right)\right\|:=\sqrt{\left\|y^{*}\right\|^{2}+\left\|y^{* *}\right\|^{2}}$.

Now let $S: E \rightrightarrows E^{*}$. We write $G(S)$ for the graph of $S, D(S)$ for the domain of $S$ and $R(S)$ for the range of $S$. We will always suppose that $G(S) \neq \emptyset$ (equivalently, $D(S) \neq \emptyset$ or $R(S) \neq \emptyset$ ). We say that $S$ is closed if $G(S)$ is closed.

Definition 2.1. We say that $S$ is quasidense if, for all $\left(x, x^{*}\right) \in E \times E^{*}$,

$$
\inf _{\left(s, s^{*}\right) \in G(S)}\left[\frac{1}{2}\|s-x\|^{2}+\frac{1}{2}\left\|s^{*}-x^{*}\right\|^{2}+\left\langle s-x, s^{*}-x^{*}\right\rangle\right] \leq 0 .
$$


See [23, Example 7.1, eqn. (28), p. 1031]. Quasidensity is actually a special case of the more general concept of $r_{L}$-density considered in [23, Section 4].

Theorem 2.2 (Quasidensity and maximality). Let $S: E \rightrightarrows E^{*}$ be closed, monotone and quasidense. Then $S$ is maximally monotone.

Proof. Let $\left(x, x^{*}\right) \in E \times E^{*}, G(S) \cup\left\{\left(x, x^{*}\right)\right\}$ be monotone and $\varepsilon>0$. By hypothesis, there exists $\left(s, s^{*}\right) \in G(S)$ such that

$$
\frac{1}{2}\|s-x\|^{2}+\frac{1}{2}\left\|s^{*}-x^{*}\right\|^{2}+\left\langle s-x, s^{*}-x^{*}\right\rangle<\varepsilon .
$$

Since $G(S) \cup\left\{\left(x, x^{*}\right)\right\}$ is monotone, $\left\langle s-x, s^{*}-x^{*}\right\rangle \geq 0$. Consequently, $\frac{1}{2}\|s-x\|^{2}+\frac{1}{2}\left\|s^{*}-x^{*}\right\|^{2}<\varepsilon$. However, $G(S)$ is closed: thus, letting $\varepsilon \rightarrow 0$, $\left(x, x^{*}\right) \in G(S)$. This completes the proof of the maximality of $S$. (This proof is adapted from that of [23, Lemma 4.7, p. 1027] — the result appears explicitly in [23, Theorem 7.4(a), pp. 1032-1033].)

Example 2.3 (The tail operator). Let $E=\ell_{1}$, and define the linear map $T: \ell_{1} \rightarrow \ell_{\infty}=E^{*}$ by $(T x)_{n}=\sum_{k \geq n} x_{k}$. It is well known that $T$ is maximally monotone. However, $T$ is not quasidense: see [23, Example 7.10, pp. 1034-1035].

Definition 2.4. We define $\left.\left.\varphi_{S}: E \times E^{*} \rightarrow\right]-\infty, \infty\right]$ by

$$
\varphi_{S}\left(x, x^{*}\right):=\sup _{\left(s, s^{*}\right) \in G(S)}\left[\left\langle s, x^{*}\right\rangle+\left\langle x, s^{*}\right\rangle-\left\langle s, s^{*}\right\rangle\right] .
$$

Lemma 2.5. Let $S: E \rightrightarrows E^{*}$ be closed, monotone and quasidense. Then:

$$
\left.\begin{array}{l}
\text { for all }\left(x, x^{*}\right) \in E \times E^{*}, \varphi_{S}\left(x, x^{*}\right) \geq\left\langle x, x^{*}\right\rangle \quad \text { and } \\
\left\{\left(x, x^{*}\right) \in E \times E^{*}: \varphi_{S}\left(x, x^{*}\right)=\left\langle x, x^{*}\right\rangle\right\}=G(S) .
\end{array}\right\}
$$

Proof. See [23, Lemma 7.7, eqn. (30), p. 1034].

The next result is somewhat subtler, and will be used in Section 3 ,

Lemma 2.6. Let $S: E \rightrightarrows E^{*}$ be closed, monotone and quasidense. Then:

$$
\begin{gathered}
\text { for all }\left(x, x^{*}\right) \in E \times E^{*}, \varphi_{S}{ }^{*}\left(x^{*}, \widehat{x}\right) \geq\left\langle x, x^{*}\right\rangle \quad \text { and } \\
\left\{\left(x, x^{*}\right) \in E \times E^{*}: \varphi_{S}{ }^{*}\left(x^{*}, \widehat{x}\right)=\left\langle x, x^{*}\right\rangle\right\}=G(S) .
\end{gathered}
$$

Proof. See [23, Lemma 7.7, eqn. (33), p. 1034]. $\left(\varphi_{S}^{*}\left(x^{*}, \widehat{x}\right)\right.$ is denoted by $\varphi_{S}{ }^{\circledR}\left(x, x^{*}\right)$ in [23]. See [23, Definition 3.1].)

In Theorem 2.7, we show that the function $\varphi_{S}$ can be used to give a simple criterion for quasidensity. We will give more criteria in Theorems 6.1 6.2 and 8.3 and Remark 8.4

Theorem 2.7. Let $S: E \rightrightarrows E^{*}$ be maximally monotone. Then $S$ is quasidense if, and only if, for all $\left(y^{*}, y^{* *}\right) \in E^{*} \times E^{* *}, \varphi_{S}{ }^{*}\left(y^{*}, y^{* *}\right) \geq\left\langle y^{*}, y^{* *}\right\rangle$.

Proof. See [23, Corollary 6.4, p. 1029-1030] for a more general result. 
Remark 2.8. We note that (2.1) is true even if $S$ is merely maximally monotone. (This was actually proved by Fitzpatrick in 4.) On the other hand, Theorem 2.7 gives a criterion for quasidensity.

Theorem 2.14 below is a very important result. By virtue of Theorem 2.2 it generalizes Rockafellar's result that subdifferentials are maximally monotone. The proof of Lemma 2.13 uses two basic results from convex analysis. The first of these, Lemma 2.9, is the Brøndsted-Rockafellar theorem, which was first proved in [3, p. 608]. There are many variations of this result: we will use Corollary 2.10, which appeared explicitly in [17, Theorem 3.3, p. 1380] (if not before). The second result from convex analysis that we will use appears in Lemma 2.12 This follows from Rockafellar's formula for the subdifferential of a sum. See [15, Theorem 3(a), pp. 85-86].

Lemma 2.9. Let $h \in \mathcal{P C} \mathcal{L S C}(E), \inf _{E} h>-\infty, \alpha, \beta>0, u \in \operatorname{dom} h$ and $h(u)<\inf _{E} h+\alpha \beta$. Then there exists $\left(s, x^{*}\right) \in G(\partial h)$ such that $h(s) \leq h(u)$, $\|s-u\| \leq \alpha$ and $\left\|x^{*}\right\| \leq \beta$.

Corollary 2.10. Let $h \in \mathcal{P C} \mathcal{L S C}(E), \inf _{E} h>-\infty$ and $\beta>0$. Then there exists $\left(s, x^{*}\right) \in G(\partial h)$ such that $h(s) \leq \inf _{E} h+\beta$ and $\left\|x^{*}\right\| \leq \beta$.

Proof. We can choose $u \in E$ such that $h(u) \leq \inf _{E} h+\beta$. The result follows from Lemma 2.9 with $\alpha=1$.

Remark 2.11. We note that Corollary 2.10 can be put in the following form: Let $h \in \mathcal{P C} \mathcal{L S C}(E)$ and $\inf _{E} h>-\infty$. Then there exists a sequence $\left\{\left(s_{n}, x_{n}^{*}\right)\right\}$ of element of $G(\partial h)$ such that $\left(h\left(s_{n}, x_{n}^{*}\right), x_{n}^{*}\right) \rightarrow\left(\inf _{E} h, 0\right)$.

Lemma 2.12. Let $g: E \rightarrow]-\infty, \infty]$ be proper and convex and $k: E \rightarrow \mathbb{R}$ be convex and continuous. Then, for all $x \in E, \partial(g+k)(x)=\partial g(x)+\partial k(x)$.

Lemma 2.13. Let $g \in \mathcal{P C} \mathcal{L S C}(E)$ and $\varepsilon>0$. Then there exists $\left(s, s^{*}\right) \in G(\partial g)$ such that $\frac{1}{2}\|s\|^{2}+\left\langle s, s^{*}\right\rangle+\frac{1}{2}\left\|s^{*}\right\|^{2}<\varepsilon$.

Proof. It is well known (from a separation theorem in $E \times E^{*}$ ) that $g$ dominates a continuous affine function. Thus there exist $\gamma_{0}, \delta_{0} \in \mathbb{R}$ such that, for all $x \in E$, $g(x) \geq-\gamma_{0}\|x\|-\delta_{0}$. Let $j(x):=\frac{1}{2}\|x\|^{2}$. Then, for all $x \in E$,

$$
(g+j)(x) \geq \frac{1}{2}\|x\|^{2}-\gamma_{0}\|x\|-\delta_{0} \geq \min _{\lambda \in \mathbb{R}}\left[\frac{1}{2} \lambda^{2}-\gamma_{0} \lambda-\delta_{0}\right] \in \mathbb{R} .
$$

Let $m:=\inf _{E}(g+j)>-\infty$. By completing the square, there exists $M \in[0, \infty[$ such that

$$
\frac{1}{2} \lambda^{2}-\gamma_{0} \lambda-\delta_{0} \leq m+1 \quad \Longrightarrow \quad \lambda \leq M .
$$

Choose $\beta \in] 0,1]$ such that $2 M \beta+\frac{1}{2} \beta^{2}<\varepsilon$. From Corollary 2.10, there exists $\left(s, x^{*}\right) \in G(\partial(g+j))$ such $(g+j)(s) \leq m+\beta \leq m+1$, and $\left\|x^{*}\right\| \leq \beta$. (2.2) implies that $\frac{1}{2}\|s\|^{2}-\gamma_{0}\|s\|-\delta_{0} \leq m+1$, and so (2.3) gives

$$
\|s\| \leq M
$$


From Lemma 2.12 $\partial(g+j)(s)=\partial g(s)+\partial j(s)$, so there exists $s^{*} \in \partial g(s)$ such that $x^{*}-s^{*} \in \partial j(s)$, from which

$$
\frac{1}{2}\|s\|^{2}=\left\langle s, x^{*}-s^{*}\right\rangle-\frac{1}{2}\left\|s^{*}-x^{*}\right\|^{2} \quad \text { and } \quad\left\|s^{*}-x^{*}\right\|=\|s\| .
$$

Thus, since $\left\|x^{*}\right\| \leq \beta,\left\|s^{*}\right\| \leq\|s\|+\beta$, and so

$$
\begin{aligned}
\frac{1}{2}\|s\|^{2}+\left\langle s, s^{*}\right\rangle+\frac{1}{2}\left\|s^{*}\right\|^{2} & =\left\langle s, x^{*}\right\rangle-\frac{1}{2}\left\|s^{*}-x^{*}\right\|^{2}+\frac{1}{2}\left\|s^{*}\right\|^{2} \\
& \leq\|s\| \beta-\frac{1}{2}\|s\|^{2}+\frac{1}{2}(\|s\|+\beta)^{2} \leq 2\|s\| \beta+\frac{1}{2} \beta^{2} .
\end{aligned}
$$

From (2.4), $\frac{1}{2}\|s\|^{2}+\left\langle s, s^{*}\right\rangle+\frac{1}{2}\left\|s^{*}\right\|^{2} \leq 2 M \beta+\frac{1}{2} \beta^{2}<\varepsilon$.

Theorem 2.14. Let $f \in \mathcal{P C} \mathcal{L S C}(E)$. Then $\partial f$ is closed, monotone and quasidense.

Proof. Let $\left(x, x^{*}\right) \in E \times E^{*}$. We apply Lemma 2.13 to the function $g:=f(\cdot+x)-x^{*}$, and the result follows since $G(\partial g)=G(\partial f)-\left(x, x^{*}\right)$.

Remark 2.15. Another proof of Theorem 2.14 can be found in 23, Theorem 7.5, p. 1033], using [23, Theorem 5.2] and [23, Corollary 4.5]. The proof given here is a simplified version of that given in [22, Theorem 8.4]. This result was extended to nonconvex functions in [25, Theorem 3.2, pp. 634-635] (using an appropriate definition of subdifferential for a nonconvex function).

We now give two simple but significant applications of Theorem 2.14.

Example 2.16. Let $\widetilde{K}$ be a nonempty $w\left(E^{*}, E\right)$-compact convex subset of $E^{*}$. We define the continuous sublinear functional $\tau_{\widetilde{K}}$ on $E$ by $\tau_{\widetilde{K}}:=\max \langle\cdot, \widetilde{K}\rangle$. From Theorem 2.14].

$$
\partial \tau_{\widetilde{K}} \text { is closed, monotone and quasidense. }
$$

By direct computation, $\tau_{\widetilde{K}}^{*}=\mathbb{I}_{\widetilde{K}}$, where $\mathbb{I}_{\widetilde{K}}\left(x^{*}\right)=0$ if $x^{*} \in \widetilde{K}$ and $\mathbb{I}_{\widetilde{K}}\left(x^{*}\right)=\infty$ if $x^{*} \in E^{*} \backslash \widetilde{K}$, and so

$$
\left.\begin{array}{rl}
x^{*} \in \partial \tau_{\widetilde{K}}(x) & \Longleftrightarrow \tau_{\widetilde{K}}(x)+\mathbb{I}_{\widetilde{K}}\left(x^{*}\right)=\left\langle x, x^{*}\right\rangle \\
& \Longleftrightarrow x^{*} \in \widetilde{K} \text { and }\left\langle x, x^{*}\right\rangle=\max \langle x, \widetilde{K}\rangle .
\end{array}\right\}
$$

Using the $w\left(E^{*}, E\right)$-compactness of $\widetilde{K}$, this implies that $D\left(\partial \tau_{\widetilde{K}}\right)=E$ and $R\left(\partial \tau_{\widetilde{K}}\right) \subset \widetilde{K}$. On the other hand, if $x^{*} \in \widetilde{K}$ then $x^{*} \in \partial \tau_{\widetilde{K}}(0)$. To sum up:

$$
D\left(\partial \tau_{\widetilde{K}}\right)=E \quad \text { and } \quad R\left(\partial \tau_{\widetilde{K}}\right)=\widetilde{K} .
$$

Example 2.17. Let $K$ be a nonempty bounded closed convex subset of $E$ and $\mathbb{I}_{K}(x)=0$ if $x \in K$ and $\mathbb{I}_{K}(x)=\infty$ if $x \in E \backslash K$. From Theorem 2.14.

$\partial \mathbb{I}_{K}$ is closed, monotone and quasidense. 
We define the continuous, sublinear functional $\sigma_{K}$ on $E^{*}$ by $\sigma_{K}:=\sup \langle K, \cdot\rangle$. By direct computation, $\mathbb{I}_{K}{ }^{*}=\sigma_{K}$, and so

$$
\left.\begin{array}{rl}
x^{*} \in \partial \mathbb{I}_{K}(x) & \Longleftrightarrow \mathbb{I}_{K}(x)+\sigma_{K}\left(x^{*}\right)=\left\langle x, x^{*}\right\rangle \\
& \Longleftrightarrow x \in K \text { and }\left\langle x, x^{*}\right\rangle=\sup \left\langle K, x^{*}\right\rangle .
\end{array}\right\}
$$

$\left(\partial \mathbb{I}_{K}\right.$ is the normal cone multifunction of $K$.) (2.9) clearly implies that $D\left(\partial \mathbb{I}_{K}\right) \subset K$. On the other hand, if $x \in K$ then $0 \in \partial \mathbb{I}_{K}(x)$. Consequently,

$$
D\left(\partial \mathbb{I}_{K}\right)=K .
$$

Now suppose, in addition, that $K$ is $w\left(E, E^{*}\right)$-compact. From (2.9),

$$
R\left(\partial \mathbb{I}_{K}\right)=E^{*} .
$$

Let $\widehat{K}:=\{\widehat{x}: x \in K\}$. It is easy to see that $\widehat{K}$ is $w\left(E^{* *}, E^{*}\right)$-closed, from which

$$
\sigma_{K}{ }^{*}=\mathbb{I}_{\widehat{K}} .
$$

\section{The Fitzpatrick extension}

Definition 3.1 (The Fitzpatrick extension). (See [23, Definition 8.5, p. 1037].) Let the notation be as in Section 2 and $S: E \rightrightarrows E^{*}$ be closed, monotone and quasidense. From Theorem 2.7 for all $\left(y^{*}, y^{* *}\right) \in E^{*} \times E^{* *}, \varphi_{S}{ }^{*}\left(y^{*}, y^{* *}\right) \geq$ $\left\langle y^{*}, y^{* *}\right\rangle$. We define $S^{\mathbb{F}}: E^{*} \rightrightarrows E^{* *}$ so that

$$
\left(y^{*}, y^{* *}\right) \in G\left(S^{\mathbb{F}}\right) \text { exactly when } \varphi_{S}{ }^{*}\left(y^{*}, y^{* *}\right)=\left\langle y^{*}, y^{* *}\right\rangle .
$$

It is easily seen that $S^{\mathbb{F}}$ is monotone. Then, from Lemma 2.6

$$
\left(x, x^{*}\right) \in G(S) \Longleftrightarrow\left(x^{*}, \widehat{x}\right) \in G\left(S^{\mathbb{F}}\right),
$$

and so $S^{\mathbb{F}}$ is, in some sense, an extension of $S$. We will describe $S^{\mathbb{F}}$ as the Fitzpatrick extension of $S$. (We note that $\Phi_{G(S)}=\varphi_{S}$ in 23 .)

Following the notation introduced in [23, Example 7.1, p. 1031], we define the map $L: E \times E^{*} \rightarrow E^{*} \times E^{* *}$ by $L\left(x, x^{*}\right):=\left(x^{*}, \widehat{x}\right)$.

Lemma 3.2. Let $S: E \rightrightarrows E^{*}$ be closed, monotone and quasidense and $R\left(S^{\mathbb{F}}\right) \subset \widehat{E}:=\{\widehat{x}: x \in E\}$. Then $G\left(S^{\mathbb{F}}\right)=L(G(S))$.

Proof. If $\left(y^{*}, y^{* *}\right) \in G\left(S^{\mathbb{F}}\right)$ then $y^{* *} \in R\left(S^{\mathbb{F}}\right) \subset \widehat{E}$, and so there exists $x \in E$ such that $\widehat{x}=y^{* *}$. Thus $\left(y^{*}, \widehat{x}\right) \in G\left(S^{\mathbb{F}}\right)$. From (3.2), $\left(x, y^{*}\right) \in G(S)$. Since $\left(y^{*}, y^{* *}\right)=\left(y^{*}, \widehat{x}\right)=L\left(x, y^{*}\right)$, this establishes that $G\left(S^{\mathbb{F}}\right) \subset L(G(S))$. On the other hand, if $\left(y^{*}, y^{* *}\right) \in L(G(S))$ then there exists $\left(x, x^{*}\right) \in G(S)$ such that $\left(y^{*}, y^{* *}\right)=\left(x^{*}, \widehat{x}\right)$. From (3.2), $\left(x^{*}, \widehat{x}\right) \in G\left(S^{\mathbb{F}}\right)$, that is to say $\left(y^{*}, y^{* *}\right) \in G\left(S^{\mathbb{F}}\right)$. Thus we have proved that $L(G(S)) \subset G\left(S^{\mathbb{F}}\right)$. 
Lemma 3.3. Let $f \in \mathcal{P C} \mathcal{L S C}(E)$. Then $G\left((\partial f)^{\mathbb{F}}\right) \subset G\left(\partial\left(f^{*}\right)\right)$.

Proof. Let $\left(x, x^{*}\right) \in E \times E^{*}$. Then, from Definition 2.4 the definition of $\partial f$ and the Fenchel-Young inequality,

$$
\begin{aligned}
\varphi_{\partial f}\left(x, x^{*}\right) & =\sup _{\left(s, s^{*}\right) \in G(\partial f)}\left[\left\langle x, s^{*}\right\rangle+\left\langle s, x^{*}\right\rangle-\left\langle s, s^{*}\right\rangle\right] \\
& =\sup _{\left(s, s^{*}\right) \in G(\partial f)}\left[\left\langle x, s^{*}\right\rangle+\left\langle s, x^{*}\right\rangle-f(s)-f^{*}\left(s^{*}\right)\right] \\
& \leq \sup _{s^{*} \in E^{*}}\left[\left\langle x, s^{*}\right\rangle-f^{*}\left(s^{*}\right)\right]+\sup _{s \in E}\left[\left\langle s, x^{*}\right\rangle-f(s)\right] \\
& \leq f(x)+f^{*}\left(x^{*}\right) .
\end{aligned}
$$

Consequently, for all $\left(y^{*}, y^{* *}\right) \in E^{*} \times E^{* *}$,

$$
\begin{aligned}
\varphi_{\partial f}^{*}\left(y^{*}, y^{* *}\right) & =\sup _{\left(x, x^{*}\right) \in E \times E^{*}}\left[\left\langle x, y^{*}\right\rangle+\left\langle x^{*}, y^{* *}\right\rangle-\varphi_{\partial f}\left(x, x^{*}\right)\right] \\
& \geq \sup _{\left(x, x^{*}\right) \in E \times E^{*}}\left[\left\langle x, y^{*}\right\rangle+\left\langle x^{*}, y^{* *}\right\rangle-f(x)-f^{*}\left(x^{*}\right)\right] \\
& =\sup _{x \in E}\left[\left\langle x, y^{*}\right\rangle-f(x)\right]+\sup _{x^{*} \in E^{*}}\left[\left\langle x^{*}, y^{* *}\right\rangle-f^{*}\left(x^{*}\right)\right] \\
& =f^{*}\left(y^{*}\right)+f^{* *}\left(y^{* *}\right) \geq\left\langle y^{*}, y^{* *}\right\rangle .
\end{aligned}
$$

The result now follows from (3.1).

Lemma 3.4. Let $\widetilde{K}$ be a nonempty $w\left(E^{*}, E\right)$-compact convex subset of $E^{*}$ and $y^{* *} \in \partial \tau_{\widetilde{K}}^{\mathbb{F}}\left(y^{*}\right)$. Then $y^{*} \in \widetilde{K}$ and $\left\langle y^{*}, y^{* *}\right\rangle=\sup \left\langle\widetilde{K}, y^{* *}\right\rangle$.

Proof. As we observed in Example 2.16, $\tau_{\widetilde{K}}^{*}=\mathbb{I}_{\widetilde{K}}$ and so, from Lemma 3.3 , $y^{* *} \in \partial \mathbb{I}_{\widetilde{K}}\left(y^{*}\right)$, that is to say $\mathbb{I}_{\widetilde{K}}\left(y^{*}\right)+\mathbb{I}_{\widetilde{K}} \widetilde{K}^{*}\left(y^{* *}\right)=\left\langle y^{*}, y^{* *}\right\rangle$. This gives the desired result.

Lemma 3.5. Let $K$ be a nonempty $w\left(E, E^{*}\right)$-compact convex subset of $E$.

(a) Let $y^{* *} \in \partial \mathbb{I}_{K}{ }^{\mathbb{F}}\left(y^{*}\right)$. Then $y^{* *} \in \widehat{K}$ and $\left\langle y^{*}, y^{* *}\right\rangle=\sup \left\langle K, y^{*}\right\rangle$.

(b) $R\left(\partial \mathbb{I}_{K}{ }^{\mathbb{F}}\right) \subset \widehat{K} \subset \widehat{E}$.

Proof. (a) As we observed in Example 2.17, $\mathbb{I}_{K}{ }^{*}=\sigma_{K}$ and so, from Lemma 3.3 $y^{* *} \in \partial \sigma_{K}\left(y^{*}\right)$, that is to say, $\sigma_{K}\left(y^{*}\right)+\sigma_{K}{ }^{*}\left(y^{* *}\right)=\left\langle y^{*}, y^{* *}\right\rangle$. From (2.12), $\sigma_{K}\left(y^{*}\right)+\mathbb{I}_{\widehat{K}}\left(y^{* *}\right)=\left\langle y^{*}, y^{* *}\right\rangle$, which gives (a). (b) is immediate from (a).

Theorem 3.6. Let $S: E \rightrightarrows E^{*}$ be closed, monotone and quasidense. Then $S^{\mathbb{F}}: E^{*} \rightrightarrows E^{* *}$ is maximally monotone.

Proof. See [23, Lemma 12.5, p. 1047]. There is also a sketch of a proof in Appendix 1, Section 9 .

We end this section by calculating the Fitzpatrick extension of a general subdifferential, as well as computing the Fitzpatrick extensions of the two closed, monotone, quasidense multifunctions introduced in Examples 2.16 and 2.17

Theorem 3.7. Let $f \in \mathcal{P C} \mathcal{L S C}(E)$. Then $(\partial f)^{\mathbb{F}}=\partial\left(f^{*}\right)$.

Proof. This is immediate from Lemma 3.3 and Theorem 3.6 . 
Theorem 3.8 (The Fitzpatrick extension of $\left.\partial \tau_{\widetilde{K}}\right)$. Let $\widetilde{K}$ be a nonempty $w\left(E^{*}, E\right)-$ compact convex subset of $E^{*}$. Then

$$
\left(y^{*}, y^{* *}\right) \in G\left(\partial \tau_{\widetilde{K}}^{\mathbb{F}}\right) \Longleftrightarrow y^{*} \in \widetilde{K} \text { and }\left\langle y^{*}, y^{* *}\right\rangle=\sup \left\langle\widetilde{K}, y^{* *}\right\rangle .
$$

Proof. As we observed in Example 2.16] $\tau_{\widetilde{K}}{ }^{*}=\mathbb{I}_{\widetilde{K}}$ and so, from Theorem 3.7 $\partial \tau_{\widetilde{K}}^{\mathbb{F}}=\partial \mathbb{I}_{\widetilde{K}}$. The result now follows by using the technique of Lemma 3.4

Theorem 3.9 (The Fitzpatrick extension of $\left.\partial \mathbb{I}_{K}\right)$. Let $K$ be a nonempty $w\left(E, E^{*}\right)$ compact convex subset of $E$. Then

$$
\left(y^{*}, y^{* *}\right) \in G\left(\partial \mathbb{I}_{K}^{\mathbb{F}}\right) \Longleftrightarrow y^{* *} \in \widehat{K} \text { and }\left\langle y^{*}, y^{* *}\right\rangle=\sup \left\langle K, y^{*}\right\rangle .
$$

Proof. As we observed in Example 2.17, $\mathbb{I}_{K}{ }^{*}=\sigma_{K}$ and so, from Theorem 3.7 $\partial \mathbb{I}_{K}{ }^{\mathbb{F}}=\partial \sigma_{K}$. The result now follows by using the technique of Lemma 3.5.

Remark 3.10. 23, Theorem 12.4(a), p. 1047] implies that if $S: E \rightrightarrows E^{*}$ is closed, monotone and quasidense and $\left(y^{*}, y^{* *}\right) \in E^{*} \times E^{* *}$ then

$$
\left(y^{*}, y^{* *}\right) \in G\left(S^{\mathbb{F}}\right) \Longleftrightarrow \inf _{\left(s, s^{*}\right) \in G(S)}\left\langle s^{*}-y^{*}, \widehat{s}-y^{* *}\right\rangle=0 .
$$

This is equivalent to the result proved in (9.3). There are more characterizations of $S^{\mathbb{F}}$ in Theorem 7.5 and (9.4). We do not know if $S^{\mathbb{F}}: E^{*} \rightrightarrows E^{* *}$ is necessarily quasidense in the context of Theorem [3.6. but Theorems 3.7 and 2.14 show that if $f \in \mathcal{P C} \mathcal{L S C}(E)$ then $(\partial f)^{\mathbb{F}}$ is quasidense. Theorem 3.7 is equivalent to [8. Théorème 3.1, pp. 376-378].

Lemma 3.3 is the "easy half" of Theorem 3.7 Theorem 3.7 and its two consequences, Theorems 3.8 and 3.9. will not be used any more in this paper, while Lemma 3.4 will be used in Theorem 8.3 and Lemma 3.5 will be used in Theorem 6.2. We have separated the proof of Theorem 3.7 into these two parts so that the reader is not obliged to wade through the complexities of the later part of 23] or Appendix 1, Section 9] to understand the logic of the rest of this paper. This raises the issue of finding a simple, direct proof of the inclusion $G\left(\partial\left(f^{*}\right)\right) \subset G\left((\partial f)^{\mathbb{F}}\right)$ in the context of Theorem 3.7. To date, we have not found such a proof.

\section{Two sum theorems}

The proofs of the two results in this section use, among other things, the bivariate version of the Attouch-Brezis theorem first proved in Simons-Zălinescu [24, Section 4, pp. 8-10]. Theorem 4.1 will be used in Theorems 5.2 and 6.1, while Theorem 4.2 will be used in Theorems 6.2 and 8.3

Theorem 4.1 (Sum theorem with domain constraints). Let $S, T: E \rightrightarrows E^{*}$ be closed, monotone and quasidense and either $D(S) \cap \operatorname{int} D(T) \neq \emptyset$ or int $D(S) \cap D(T) \neq \emptyset$. Then $S+T$ is closed, monotone and quasidense.

Proof. See [23, Theorem 8.4(a) $\Longrightarrow(\mathrm{d})$, pp. 1036-1037]. 
Theorem 4.2 (Sum theorem with range constraints). Let $S, T: E \rightrightarrows E^{*}$ be closed, monotone and quasidense and either $R(S) \cap \operatorname{int} R(T) \neq \emptyset$ or int $R(S) \cap R(T) \neq \emptyset$. Then the multifunction $y \mapsto\left(S^{\mathbb{F}}+T^{\mathbb{F}}\right)^{-1}(\widehat{y})$ is closed, monotone and quasidense. If, further, $R\left(T^{\mathbb{F}}\right) \subset \widehat{E}$, then the parallel sum $S \| T:=\left(S^{-1}+T^{-1}\right)^{-1}$ is closed, monotone and quasidense.

Proof. The first observation was established in [23, Theorem 8.8, p. 1039]. If $R\left(T^{\mathbb{F}}\right) \subset \widehat{E}$ then, from Lemma $3.2 G\left(T^{\mathbb{F}}\right)=L(G(T))$. Now in 23, Definition 8.5, eqn. (46), p. 1037], what was actually defined was the Fitzpatrick extension of a subset of $E \times E^{*}$ rather than that of a multifunction from $E$ into $E^{*}$, and the relation between them is $G\left(T^{\mathbb{F}}\right)=G(T)^{\mathbb{F}}$. Thus $G(T)^{\mathbb{F}}=L(G(T))$, and it now follows from [23, Theorem 8.8] that $S \| T$ is closed, monotone and quasidense.

\section{Type (FPV)}

Definition 5.1. Let $S: E \rightrightarrows E^{*}$ be monotone. We say that $S$ is of type (FPV) or maximally monotone locally if whenever $U$ is an open convex subset of $E$, $U \cap D(S) \neq \emptyset,\left(w, w^{*}\right) \in U \times E^{*}$ and

$$
\left(s, s^{*}\right) \in G(S) \quad \text { and } \quad s \in U \quad \Longrightarrow \quad\left\langle s-w, s^{*}-w^{*}\right\rangle \geq 0,
$$

then $\left(w, w^{*}\right) \in G(S)$. (If we take $U=E$, we see that every monotone multifunction of type (FPV) is maximally monotone.)

Theorem 5.2. Let $S: E \rightrightarrows E^{*}$ be closed, monotone and quasidense. Then $S$ is maximally monotone of type (FPV).

Proof. Let $U$ be an open convex subset of $E, U \cap D(S) \neq \emptyset,\left(w, w^{*}\right) \in U \times E^{*}$ and (5.1) be satisfied. Let $y \in U \cap D(S)$. Since the segment $[w, y]$ is a compact subset of the open set $U$, we can choose $\varepsilon>0$ so that $K:=[w, y]+\varepsilon E_{1} \subset U$. K is bounded, closed and convex. Let $T:=\partial \mathbb{I}_{K}$. From (2.10), $D(S) \cap \operatorname{int} D(T)=$ $D(S) \cap \operatorname{int} K \ni y$, and (2.8) and Theorem 4.1 imply that $S+T$ is closed and quasidense. Let $\eta>0$. Then there exists $\left(s, u^{*}\right) \in G(S+T)$ such that

$$
\frac{1}{2}\|s-w\|^{2}+\frac{1}{2}\left\|u^{*}-w^{*}\right\|^{2}+\left\langle s-w, u^{*}-w^{*}\right\rangle<\eta .
$$

We can choose $s^{*} \in S(s)$ and $x^{*} \in T(s)$ such that $s^{*}+x^{*}=u^{*}$. Since $s \in D(T)=K \subset U$, (5.1) implies that $\left\langle s-w, s^{*}-w^{*}\right\rangle \geq 0$ and, since $w \in K$, (2.9) implies that $\left\langle s, x^{*}\right\rangle=\sup \left\langle K, x^{*}\right\rangle \geq\left\langle w, x^{*}\right\rangle$. Combining together these two inequalities, we have $\left\langle s-w, u^{*}-w^{*}\right\rangle=\left\langle s-w, s^{*}-w^{*}\right\rangle+\left\langle s, x^{*}\right\rangle-\left\langle w, x^{*}\right\rangle \geq 0$. From (5.2),

$$
\frac{1}{2}\|s-w\|^{2}+\frac{1}{2}\left\|u^{*}-w^{*}\right\|^{2}<\eta .
$$

Thus, taking $\eta$ arbitrarily small and using the fact that $S+T$ is closed, $w^{*} \in(S+T)(w)$, from which there exist $s_{0}^{*} \in S(w)$ and $x_{0}^{*} \in T(w)$ such that $s_{0}^{*}+x_{0}^{*}=w^{*}$. From (2.9), $\left\langle w, x_{0}^{*}\right\rangle=\sup \left\langle K, x_{0}^{*}\right\rangle$. Since $w \in \operatorname{int} K, x_{0}^{*}=0$, from which $s_{0}^{*}=w^{*}$. Thus $\left(w, w^{*}\right)=\left(w, s_{0}^{*}\right) \in G(S)$, as required. 
Remark 5.3. We do not know of an example of a maximally monotone multifunction that is not of type (FPV). The tail operator (see Example 2.3) does not provide an example because it was proved in Fitzpatrick-Phelps, [6. Theorem 3.10, p. 68] that if $S: E \rightrightarrows E^{*}$ is maximally monotone and $D(S)=E$ then $S$ is of type (FPV). This question is closely related to the sum problem. (See [20, Theorem 44.1, p. 170].) Theorem [5.2 can also be deduced from Voisei-Zălinescu, [27, Remark 3.6, p. 1024].

\section{$6 \quad$ Fuzzy criteria for quasidensity}

Let $S: E \rightrightarrows E^{*}$ be closed and monotone. From Definition 2.1 $S$ is quasidense if, and only if, for all $\left(w, w^{*}\right) \in E \times E^{*}$ and $\eta>0$, there exists $\left(s, s^{*}\right) \in G(S)$ such that $\frac{1}{2}\|s-w\|^{2}+\frac{1}{2}\left\|s^{*}-w^{*}\right\|^{2}+\left\langle s-w, s^{*}-w^{*}\right\rangle<\eta$. In Theorem 6.1 we show that this is equivalent to a formally much stronger condition in which $w^{*}$ is replaced by any nonempty $w\left(E^{*}, E\right)$-compact convex subset of $E^{*}$ and, in Theorem 6.2, we show that this is equivalent to a formally much stronger condition in which $w$ is replaced by any nonempty $w\left(E, E^{*}\right)$-compact convex subset of $E$. Theorem 6.1 and Theorem 6.2 lead to Theorem 6.5, in which we prove that every closed, monotone quasidense multifunction is strongly maximal.

Theorem 6.1 (A criterion for quasidensity in which $w^{*}$ becomes fuzzy). Let $S: E \rightrightarrows E^{*}$ be closed and monotone. Then $(\mathrm{a}) \Longleftrightarrow(\mathrm{b})$.

(a) $S$ is quasidense.

(b) For all $w \in E$, nonempty $w\left(E^{*}, E\right)$-compact convex subsets $\widetilde{W}$ of $E^{*}$ and $\eta>0$, there exists $\left(s, s^{*}\right) \in G(S)$ such that

$$
\frac{1}{2}\|s-w\|^{2}+\frac{1}{2} \operatorname{dist}\left(s^{*}, \widetilde{W}\right)^{2}+\max \left\langle s-w, s^{*}-\widetilde{W}\right\rangle<\eta .
$$

Proof. First suppose that (a) is true. Let $w \in E$ and $\widetilde{W}$ be a nonempty $w\left(E^{*}, E\right)$-compact convex subset of $E^{*}$. We define the multifunction ${ }_{w} S: E \rightrightarrows E^{*}$ so that $G\left({ }_{w} S\right)=G(S)-(w, 0)$, and write $T:=\partial \tau_{-\widetilde{W}}$. Clearly, ${ }_{w} S$ is closed, monotone and quasidense and, from (2.5) and (2.7) with $\widetilde{K}:=-\widetilde{W}$, $T$ is closed, monotone and quasidense and $D(T)=E$. Theorem 4.1 now implies that ${ }_{w} S+T$ is also closed, monotone and quasidense. Thus, for all $\eta>0$, there exist $x \in E, s^{*} \in{ }_{w} S(x)$ and $x^{*} \in T(x)$ such that

$$
\frac{1}{2}\|x\|^{2}+\frac{1}{2}\left\|s^{*}+x^{*}\right\|^{2}+\left\langle x, s^{*}+x^{*}\right\rangle<\eta .
$$

From (2.6), $x^{*} \in-\widetilde{W}$ and $\left\langle x, x^{*}\right\rangle=\max \langle x,-\widetilde{W}\rangle$, and so $\max \left\langle x, s^{*}-\widetilde{W}\right\rangle=$ $\left\langle x, s^{*}+x^{*}\right\rangle$. Since $s^{*} \in{ }_{w} S(x), s^{*} \in S(x+w)$. Let $s:=x+w$ : then $x=s-w$ and $\left(s, s^{*}\right) \in G(S)$. (b) now follows since $\left\|s^{*}+x^{*}\right\|=\left\|s^{*}-\left(-x^{*}\right)\right\| \geq \operatorname{dist}\left(s^{*}, \widetilde{W}\right)$.

Suppose, conversely, that (b) is true. Let $\left(w, w^{*}\right) \in E \times E^{*}$ and $\eta>0$. Define $\widetilde{W}:=\left\{w^{*}\right\}$, and let $\left(s, s^{*}\right)$ be as in (b). Then (b) implies that

$$
\frac{1}{2}\|s-w\|^{2}+\frac{1}{2}\left\|s^{*}-w^{*}\right\|^{2}+\left\langle s-w, s^{*}-w^{*}\right\rangle<\eta .
$$

Thus $S$ is quasidense, and so (a) is true. 
We can think of the next result as a "dual" to Theorem 6.1.

Theorem 6.2 (A criterion for quasidensity in which $w$ becomes fuzzy). Let $S: E \rightrightarrows E^{*}$ be closed and monotone. Then $(\mathrm{a}) \Longleftrightarrow(\mathrm{b})$.

(a) $S$ is quasidense.

(b) For all nonempty $w\left(E, E^{*}\right)$-compact convex subsets $W$ of $E, w^{*} \in E^{*}$ and $\eta>0$, there exists $\left(s, s^{*}\right) \in G(S)$ such that

$$
\frac{1}{2} \operatorname{dist}(s, W)^{2}+\frac{1}{2}\left\|s^{*}-w^{*}\right\|^{2}+\max \left\langle s-W, s^{*}-w^{*}\right\rangle<\eta .
$$

Proof. First suppose that (a) is true. Let $W$ be a nonempty $w\left(E, E^{*}\right)$-compact convex subset of $E$ and $w^{*} \in E^{*}$. Clearly, $S-w^{*}$ is closed, monotone and quasidense. Let $T:=\partial \mathbb{I}_{-W}$. From (2.8) and (2.11) with $K:=-W, T$ is closed, monotone and quasidense and $R(T)=E^{*}$. Lemma 3.5 (b) and Theorem 4.2 now imply that $\left(S-w^{*}\right) \| T$ is also closed, monotone and quasidense. Thus, for all $\eta>0$, there exist $x^{*} \in E^{*},\left(s, x^{*}\right) \in G\left(S-w^{*}\right)$ and $\left(x, x^{*}\right) \in G(T)$ such that

$$
\frac{1}{2}\|s+x\|^{2}+\frac{1}{2}\left\|x^{*}\right\|^{2}+\left\langle s+x, x^{*}\right\rangle<\eta .
$$

From (2.9), $x \in-W$ and $\left\langle x, x^{*}\right\rangle=\max \left\langle-W, x^{*}\right\rangle$, from which $\max \left\langle s-W, x^{*}\right\rangle=$ $\left\langle s+x, x^{*}\right\rangle$. Since $\left(s, x^{*}\right) \in G\left(S-w^{*}\right), x^{*}+w^{*} \in S(s)$. Let $s^{*}:=x^{*}+w^{*}$ : then $x^{*}=s^{*}-w^{*}$ and $\left(s, s^{*}\right) \in G(S)$. (b) now follows since $\|s+x\|=\|s-(-x)\| \geq$ $\operatorname{dist}(s, W)$.

Suppose, conversely, that (b) is true. Let $\left(w, w^{*}\right) \in E \times E^{*}$ and $\eta>0$. Define $W:=\{w\}$, and let $\left(s, s^{*}\right)$ be as in (b). Then (b) implies that

$$
\frac{1}{2}\|s-w\|^{2}+\frac{1}{2}\left\|s^{*}-w^{*}\right\|^{2}+\left\langle s-w, s^{*}-w^{*}\right\rangle<\eta .
$$

Thus $S$ is quasidense, and so (a) is true.

Theorem 6.5 below states that a closed, monotone quasidense multifunction is strongly maximal in the sense of [17. Theorems 6.1-2, pp. 1386-1387]. It is worth pointing out that we do not know of a maximally monotone multifunction that is not strongly maximal. The tail operator (see Example 2.31) does not provide an example because it was proved in Bauschke-Simons, [1, Theorem 1.1, pp. 166-167] that if $S: D(S) \subset E \rightarrow E^{*}$ is linear and maximally monotone then $S$ is strongly maximal.

We will use the computational rules contained in Lemmas 6.3 and 6.4 below. $\mathcal{T}_{E}$ stands for the norm topology of $E$ and $\mathcal{T}_{E^{*}}$ for the norm topology of $E^{*}$. We note that Lemma 6.3 is true even if $S$ is merely maximally monotone. (See Remark 2.8.)

Lemma 6.3. Let $S: E \rightrightarrows E^{*}$ be closed, monotone and quasidense, $\left\{\left(s_{\beta}, s_{\beta}^{*}\right)\right\}$ be a bounded net of elements of $G(S)$ and $\left(z, z^{*}\right) \in E \times E^{*}$.

(a) If $\left(s_{\beta}, s_{\beta}^{*}\right) \rightarrow\left(z, z^{*}\right)$ in $\mathcal{T}_{E} \times w\left(E^{*}, E\right)$ then $\left(z, z^{*}\right) \in G(S)$.

(b) If $\left(s_{\beta}, s_{\beta}^{*}\right) \rightarrow\left(z, z^{*}\right)$ in $w\left(E, E^{*}\right) \times \mathcal{T}_{E^{*}}$ then $\left(z, z^{*}\right) \in G(S)$. 
Proof. Note that $\left\langle s_{\beta}, s_{\beta}^{*}\right\rangle-\left\langle z, z^{*}\right\rangle=\left\langle s_{\beta}-z, z^{*}\right\rangle+\left\langle s_{\beta}-z, s_{\beta}^{*}-z^{*}\right\rangle+\left\langle z, s_{\beta}^{*}-z^{*}\right\rangle$. In both cases, $\lim _{\beta}\left\langle s_{\beta}-z, z^{*}\right\rangle=0$ and $\lim _{\beta}\left\langle z, s_{\beta}^{*}-z^{*}\right\rangle=0$. In case (a), we have $\lim _{\beta}\left\|s_{\beta}-z\right\|=0$ and $\sup _{\beta}\left\|s_{\beta}^{*}-z^{*}\right\|<\infty$. In case (b), we have $\sup _{\beta}\left\|s_{\beta}-z\right\|<\infty$ and $\lim _{\beta}\left\|s_{\beta}^{*}-z^{*}\right\|=0$. Thus, in both cases, we have

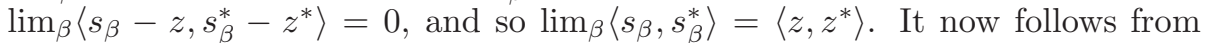
(2.1) that $\lim _{\beta} \varphi_{S}\left(s_{\beta}, s_{\beta}^{*}\right)=\left\langle z, z^{*}\right\rangle$. Since $\varphi_{S}$ is $w\left(E, E^{*}\right) \times w\left(E^{*}, E\right)$ lower semicontinuous and $\left(s_{\beta}, s_{\beta}^{*}\right) \rightarrow\left(z, z^{*}\right)$ in $w\left(E, E^{*}\right) \times w\left(E^{*}, E\right)$, it follows that $\varphi_{S}\left(z, z^{*}\right) \leq\langle z, z\rangle$, and another application of (2.1) gives $\left(z, z^{*}\right) \in G(S)$.

Lemma 6.4. (a) Let $\widetilde{W}$ be a nonempty $w\left(E^{*}, E\right)$-compact subset of $E^{*}$ and $\left\{s_{\alpha}^{*}\right\}$ be a net of elements of $E^{*}$ such that $\lim _{\alpha} \operatorname{dist}\left(s_{\alpha}^{*}, \widetilde{W}\right)=0$. Then there exist $w^{*} \in \widetilde{W}$ and a subnet $\left\{s_{\beta}^{*}\right\}$ of $\left\{s_{\alpha}^{*}\right\}$ such that $s_{\beta}^{*} \rightarrow w^{*}$ in $w\left(E^{*}, E\right)$.

(b) Let $W$ be a nonempty $w\left(E, E^{*}\right)$-compact subset of $E$ and $\left\{s_{\alpha}^{*}\right\}$ be a net of elements of $E$ such that $\lim _{\alpha} \operatorname{dist}\left(s_{\alpha}, W\right)=0$. Then there exist $w \in W$ and $a$ subnet $\left\{s_{\beta}\right\}$ of $\left\{s_{\alpha}\right\}$ such that $s_{\beta} \rightarrow w$ in $w\left(E, E^{*}\right)$.

Proof. In case (a), there exists a net $\left\{w_{\alpha}^{*}\right\}$ of elements of $\widetilde{W}$ such that $\lim _{\alpha}\left\|w_{\alpha}^{*}-s_{\alpha}^{*}\right\|=0$. Since $\widetilde{W}$ is $w\left(E^{*}, E\right)$-compact, there exist $w^{*} \in \widetilde{W}$ and a subnet $\left\{w_{\beta}^{*}\right\}$ of $\left\{w_{\alpha}^{*}\right\}$ such that $w_{\beta}^{*} \rightarrow w^{*}$ in $w\left(E^{*}, E\right)$. (a) follows since manifestly $s_{\beta}^{*} \rightarrow w^{*}$ in $w\left(E^{*}, E\right)$ also. The proof of (b) is similar.

Theorem 6.5 (Quasidensity implies "strong maximality"). Let $S: E \rightrightarrows E^{*}$ be closed, monotone and quasidense. Then, whenever $w \in E$ and $\widetilde{W}$ is a nonempty $w\left(E^{*}, E\right)$-compact convex subset of $E^{*}$ such that,

$$
\text { for all }\left(s, s^{*}\right) \in G(S), \max \left\langle s-w, s^{*}-\widetilde{W}\right\rangle \geq 0,
$$

then $S w \cap \widetilde{W} \neq \emptyset$ and, further, whenever $W$ is a nonempty $w\left(E, E^{*}\right)$-compact convex subset of $E, w^{*} \in E^{*}$ and,

$$
\text { for all }\left(s, s^{*}\right) \in G(S), \max \left\langle s-W, s^{*}-w^{*}\right\rangle \geq 0,
$$

then $w^{*} \in S(W)$.

Proof. Let $w \in E, \widetilde{W}$ be a nonempty $w\left(E^{*}, E\right)$-compact convex subset of $E^{*}$, and (6.1) be satisfied. From Theorem [6.1, for all $\eta>0$, there exists $\left(s, s^{*}\right) \in$ $G(S)$ such that

$$
\frac{1}{2}\|s-w\|^{2}+\frac{1}{2} \operatorname{dist}\left(s^{*}, \widetilde{W}\right)^{2}<\eta .
$$

Thus there exist a sequence $\left\{\left(s_{n}, s_{n}^{*}\right)\right\}_{n \geq 1}$ of elements of $G(S)$ such that $\lim _{n}\left\|s_{n}-w\right\|=0$ and $\lim _{n} \operatorname{dist}\left(s_{n}^{*}, \widetilde{W}\right)=0$. From Lemma 6.4(a), there exist $w^{*} \in \widetilde{W}$ and a subnet $\left\{\left(s_{\beta}, s_{\beta}^{*}\right)\right\}$ of $\left\{\left(s_{n}, s_{n}^{*}\right)\right\}$ such that $s_{\beta}^{*} \rightarrow w^{*}$ in $w\left(E^{*}, E\right)$. Obviously, $s_{\beta} \rightarrow w$ in $\mathcal{T}_{E}$ and so, from Lemma 6.3 (a), $\left(w, w^{*}\right) \in G(S)$. So $S w \cap \widetilde{W} \neq \emptyset$, as required.

Now let $W$ be a nonempty $w\left(E, E^{*}\right)$-compact convex subset of $E, w^{*} \in E^{*}$ and (6.2) be satisfied. From Theorem [6.2, for all $\eta>0$, there exists $\left(s, s^{*}\right) \in$ $G(S)$ such that

$$
\frac{1}{2} \operatorname{dist}(s, W)^{2}+\frac{1}{2}\left\|s^{*}-w^{*}\right\|^{2}<\eta \text {. }
$$


Thus there exists a sequence $\left\{\left(s_{n}, s_{n}^{*}\right)\right\}_{n \geq 1}$ of elements of $G(S)$ such that $\lim _{n} \operatorname{dist}\left(s_{n}, W\right)=0$ and $\lim _{n}\left\|s_{n}^{*}-w^{*}\right\|=0$. From Lemma 6.4(b), there exist $w \in W$ and a subnet $\left\{\left(s_{\beta}, s_{\beta}^{*}\right)\right\}$ of $\left\{\left(s_{n}, s_{n}^{*}\right)\right\}$ such that $s_{\beta} \rightarrow w$ in $w\left(E, E^{*}\right)$. Obviously, $s_{\beta}^{*} \rightarrow w^{*}$ in $\mathcal{T}_{E^{*}}$ and so, from Lemma 6.3(b), $\left(w, w^{*}\right) \in G(S)$. So $w^{*} \in S(W)$, as required.

\section{Sequential characterizatons of the Fitzpatrick extension}

The main result of this section, Theorem 7.5 contains three characterizations of the Fitzpatrick extension of a closed, monotone multifunction, two of them in terms of a sequence of elements from its graph. Theorem 7.5 is bootstrapped from Lemma 7.4, which depends on the three preceding lemmas, about which we now make a few comments.

The genesis for Lemmas 7.1 and 7.2 below is ultimately the sharpening by Gossez of a result established by Rockafellar in one of his proofs of the maximal monotonicity of subdifferentials (see [8, Lemma 3.1, pp. 376-377] and 16. Proposition 1, pp. 211-212]). Lemma 7.3 is a special case of a result from [23. In what follows, we write $\bigvee_{i=0}^{m} f_{i}$ for $\max \left\{f_{0}, \ldots, f_{m}\right\}$.

Lemma 7.1. Let $X$ be a nonzero Banach space, $m \geq 1, f_{0} \in \mathcal{P C} \mathcal{L S C}(X)$ and $f_{1}, \ldots, f_{m}$ be real, convex, continuous functions on $X$. Suppose that there exists $x^{* *} \in E^{* *}$ such that, for all $i=0, \ldots, m, f_{i}^{* *}\left(x^{* *}\right) \leq 0$. Then, for all $\varepsilon>0$, there exists $x \in X$ such that, for all $i=0, \ldots, m, f_{i}(x) \leq \varepsilon$.

Proof. We first observe that

$$
\inf _{X} \bigvee_{i=0}^{m} f_{i}=\left\langle 0, x^{* *}\right\rangle-\left(\bigvee_{i=0}^{m} f_{i}\right)^{*}(0) \leq\left(\bigvee_{i=0}^{m} f_{i}\right)^{* *}\left(x^{* *}\right)
$$

From [20, Corollary 45.5, p. 174] or [7, Corollary 7, p. 3558], $\left(\bigvee_{i=0}^{m} f_{i}\right)^{* *}\left(x^{* *}\right)=$ $\bigvee_{i=0}^{m} f_{i}^{* *}\left(x^{* *}\right)$ and, by hypothesis, $\bigvee_{i=0}^{m} f_{i}^{* *}\left(x^{* *}\right) \leq 0$. Thus $\inf _{X} \bigvee_{i=0}^{m} f_{i} \leq 0$ This gives the desired result.

Lemma 7.2. Let $f_{0} \in \mathcal{P C} \mathcal{L S C}\left(E \times E^{*}\right), z^{* *} \in E^{* *}, f_{0}^{* *}\left(z^{* *}, 0\right) \leq 0$ and $z^{*} \in E^{*}$. Then, for all $n \in \mathbb{N}$, there exists $\left(x_{n}, x_{n}^{*}\right) \in E \times E^{*}$ such that

$$
\begin{gathered}
f_{0}\left(x_{n}, x_{n}^{*}\right) \leq 1 / n^{2}, \\
\left\|x_{n}\right\| \leq\left\|z^{* *}\right\|+1 / n^{2},\left\|x_{n}^{*}\right\| \leq 1 / n^{2}, \text { and }\left|\left\langle x_{n}, z^{*}\right\rangle-\left\langle z^{*}, z^{* *}\right\rangle\right| \leq 1 / n^{2} .
\end{gathered}
$$

Proof. Define the real, continuous convex functions $f_{1}, f_{2}, f_{3}, f_{4}$ on $E \times E^{*}$ by $f_{1}\left(x, x^{*}\right):=\|x\|-\left\|z^{* *}\right\|, f_{2}\left(x, x^{*}\right):=\left\|x^{*}\right\|, f_{3}\left(x, x^{*}\right):=\left\langle x, z^{*}\right\rangle-\left\langle z^{*}, z^{* *}\right\rangle$ and $f_{4}\left(x, x^{*}\right):=\left\langle z^{*}, z^{* *}\right\rangle-\left\langle x, z^{*}\right\rangle$. By direct computation, for all $i=0, \ldots, 4$, $f_{i}^{* *}\left(z^{* *}, 0\right) \leq 0$ (with equality when $i=1, \ldots, 4$ ). The result now follows from Lemma 7.1 with $X=E \times E^{*}$ and $\varepsilon=1 / n^{2}$. 
Lemma 7.3. Let $g \in \mathcal{P C} \mathcal{L S C}\left(E^{*} \times E^{* *}\right)$,

$$
\left(y^{*}, y^{* *}\right) \in E^{*} \times E^{* *} \quad \Longrightarrow \quad g\left(y^{*}, y^{* *}\right) \geq\left\langle y^{*}, y^{* *}\right\rangle,
$$

$\left(z^{*}, z^{* *}\right) \in E \times E^{* *}$, and

$$
g\left(z^{*}, z^{* *}\right)=\left\langle z^{*}, z^{* *}\right\rangle
$$

Then

$$
g^{*}\left(z^{* *}, \widehat{z^{*}}\right)=\left\langle z^{*}, z^{* *}\right\rangle .
$$

Proof. Let $\left(y^{*}, y^{* *}\right) \in E^{*} \times E^{* *}$ and $\left.\lambda \in\right] 0,1[$. From (7.2), the convexity of $g$ and (7.1),

$$
\begin{aligned}
\lambda g\left(y^{*}, y^{* *}\right) & =\lambda g\left(y^{*}, y^{* *}\right)+(1-\lambda) g\left(z^{*}, z^{* *}\right)-(1-\lambda)\left\langle z^{*}, z^{* *}\right\rangle \\
& \geq g\left(\lambda y^{*}+(1-\lambda) z^{*}, \lambda y^{* *}+(1-\lambda) z^{* *}\right)-(1-\lambda)\left\langle z^{*}, z^{* *}\right\rangle \\
& \geq\left\langle\lambda y^{*}+(1-\lambda) z^{*}, \lambda y^{* *}+(1-\lambda) z^{* *}\right\rangle-(1-\lambda)\left\langle z^{*}, z^{* *}\right\rangle \\
& =\lambda^{2}\left\langle y^{*}, y^{* *}\right\rangle+\lambda(1-\lambda)\left[\left\langle y^{*}, z^{* *}\right\rangle+\left\langle z^{*}, y^{* *}\right\rangle-\left\langle z^{*}, z^{* *}\right\rangle\right] .
\end{aligned}
$$

Thus, dividing by $\lambda$,

$$
g\left(y^{*}, y^{* *}\right) \geq \lambda\left\langle y^{*}, y^{* *}\right\rangle+(1-\lambda)\left[\left\langle y^{*}, z^{* *}\right\rangle+\left\langle z^{*}, y^{* *}\right\rangle-\left\langle z^{*}, z^{* *}\right\rangle\right] .
$$

Letting $\lambda \rightarrow 0$ and rearranging the terms,

$$
\left\langle y^{*}, z^{* *}\right\rangle+\left\langle z^{*}, y^{* *}\right\rangle-g\left(y^{*}, y^{* *}\right) \leq\left\langle z^{*}, z^{* *}\right\rangle,
$$

that is to say,

$$
\left\langle\left(y^{*}, y^{* *}\right),\left(z^{* *}, \widehat{z^{*}}\right)\right\rangle-g\left(y^{*}, y^{* *}\right) \leq\left\langle z^{*}, z^{* *}\right\rangle .
$$

Taking the supremum over $\left(y^{*}, y^{* *}\right) \in E^{*} \times E^{* *}, \quad g^{*}\left(z^{* *}, \widehat{z^{*}}\right) \leq\left\langle z^{*}, z^{* *}\right\rangle . \quad$ On the other hand, from (7.2) again,

$$
\begin{aligned}
g^{*}\left(z^{* *}, \widehat{z^{*}}\right) & \geq\left\langle\left(z^{*}, z^{* *}\right),\left(z^{* *}, \widehat{z^{*}}\right)\right\rangle-g\left(z^{*}, z^{* *}\right) \\
& =2\left\langle z^{*}, z^{* *}\right\rangle-\left\langle z^{*}, z^{* *}\right\rangle=\left\langle z^{*}, z^{* *}\right\rangle .
\end{aligned}
$$

This gives (7.3), and completes the proof of Lemma 7.3 (This proof is based partly on the proof of [20, Lemma 19.12, p. 82].)

Lemma 7.4. Let $T: E \rightrightarrows E^{*}$ be closed monotone and quasidense and $\left(z^{*}, z^{* *}\right) \in E^{*} \times E^{* *}$. Then $(\mathrm{a}) \Longleftrightarrow(\mathrm{b}) \Longrightarrow(\mathrm{c}) \Longrightarrow(\mathrm{d})$ :

(a) $\left(z^{*}, z^{* *}\right) \in G\left(T^{\mathbb{F}}\right)$.

(b) $\varphi_{T}{ }^{*}\left(z^{*}, z^{* *}\right)=\left\langle z^{*}, z^{* *}\right\rangle$.

(c) $\varphi_{T}^{* *}\left(z^{* *}, z^{*}\right)=\left\langle z^{*}, z^{* *}\right\rangle$.

(d) There exists a sequence $\left\{\left(t_{n}, t_{n}^{*}\right)\right\}_{n \geq 1}$ of elements of $G(T)$ such that $\lim _{n}\left\langle t_{n}, t_{n}^{*}\right\rangle=\left\langle z^{*}, z^{* *}\right\rangle$ and $\lim _{n}\left\|t_{n}^{*}-z^{*}\right\|=0$. 
Proof. It is immediate from (3.1) that (a) $\Longleftrightarrow(\mathrm{b})$. It is also immediate from Lemma 7.3 with $g:=\varphi_{T}{ }^{*}$ and Theorem 2.7 that (b) $\Longrightarrow$ (c). Now suppose that (c) is true. Let $S:=T-z^{*}$. Clearly, $S$ is closed monotone and quasidense. It is also easily seen that $\varphi_{S}{ }^{* *}\left(z^{* *}, 0\right)=0$. From Lemma 7.2 with $f_{0}=\varphi_{S}$, for all $n \in \mathbb{N}$, there exists $\left(x_{n}, x_{n}^{*}\right) \in E \times E^{*}$ such that

$$
\begin{gathered}
\varphi_{S}\left(x_{n}, x_{n}^{*}\right) \leq 1 / n^{2}, \\
\left\|x_{n}\right\| \leq\left\|z^{* *}\right\|+1 / n^{2},\left\|x_{n}^{*}\right\| \leq 1 / n^{2}, \text { and }\left|\left\langle x_{n}, z^{*}\right\rangle-\left\langle z^{*}, z^{* *}\right\rangle\right| \leq 1 / n^{2} .
\end{gathered}
$$

From Definition 2.1 there exists $\left(s_{n}, s_{n}^{*}\right) \in G(S)$ such that

$$
\frac{1}{2}\left\|s_{n}-x_{n}\right\|^{2}+\frac{1}{2}\left\|s_{n}^{*}-x_{n}^{*}\right\|^{2}+\left\langle s_{n}-x_{n}, s_{n}^{*}-x_{n}^{*}\right\rangle \leq 1 / n^{2} .
$$

Set $M:=\left\|z^{* *}\right\|+1$. From (7.5), $\left\|x_{n}\right\| \leq M$. From (7.4) and Definition 2.4 $\left\langle s_{n}, x_{n}^{*}\right\rangle+\left\langle x_{n}, s_{n}^{*}\right\rangle-\left\langle s_{n}, s_{n}^{*}\right\rangle \leq 1 / n^{2}$. Combining this with (7.5), we see that $\left\langle s_{n}-x_{n}, s_{n}^{*}-x_{n}^{*}\right\rangle \geq\left\langle x_{n}, x_{n}^{*}\right\rangle-1 / n^{2} \geq-M / n^{2}-1 / n^{2}$, and so (7.6) gives $\frac{1}{2}\left\|s_{n}-x_{n}\right\|^{2}+\frac{1}{2}\left\|s_{n}^{*}-x_{n}^{*}\right\|^{2} \leq M / n^{2}+2 / n^{2}$. Thus

$$
\lim _{n}\left\|s_{n}-x_{n}\right\|=0 \quad \text { and } \quad \lim _{n}\left\|s_{n}^{*}-x_{n}^{*}\right\|=0 .
$$

Combining (7.7) with (7.5), $\sup _{n}\left\|s_{n}\right\|<\infty$ and $\lim _{n}\left\|s_{n}^{*}\right\|=0$, from which $\lim _{n}\left\langle s_{n}, s_{n}^{*}\right\rangle=0$. Now $\left\langle s_{n}, z^{*}\right\rangle=\left\langle s_{n}-x_{n}, z^{*}\right\rangle+\left\langle x_{n}, z^{*}\right\rangle$ and so, from (7.7) and (7.5) again, $\lim _{n}\left\langle s_{n}, z^{*}\right\rangle=\left\langle z^{*}, z^{* *}\right\rangle$. Let $\left(t_{n}, t_{n}^{*}\right):=\left(s_{n}, s_{n}^{*}+z^{*}\right) \in G(T)$. Clearly, $\lim _{n}\left\|t_{n}^{*}-z^{*}\right\|=\lim _{n}\left\|s_{n}^{*}\right\|=0$ and $\lim _{n}\left\langle t_{n}, t_{n}^{*}\right\rangle=\lim _{n}\left\langle s_{n}, s_{n}^{*}+z^{*}\right\rangle=$ $\lim _{n}\left\langle s_{n}, s_{n}^{*}\right\rangle+\lim _{n}\left\langle s_{n}, z^{*}\right\rangle=\left\langle z^{*}, z^{* *}\right\rangle$. This completes the proof of (d).

Theorem 7.5 (Sequential characterizations of the Fitzpatrick extension). Let $S: E \rightrightarrows E^{*}$ be closed monotone and quasidense and $\left(z^{*}, z^{* *}\right) \in E^{*} \times E^{* *}$. Then the following four conditions are equivalent:

(a) $\left(z^{*}, z^{* *}\right) \in G\left(S^{\mathbb{F}}\right)$.

(b) $\varphi_{S}{ }^{* *}\left(z^{* *}, \widehat{z^{*}}\right)=\left\langle z^{*}, z^{* *}\right\rangle$.

(c) For all $w^{*} \in E^{*}$, there exists a sequence $\left\{\left(s_{n}, s_{n}^{*}\right)\right\}_{n \geq 1}$ of elements of $G(S)$ such that $\left\langle s_{n}, s_{n}^{*}-w^{*}\right\rangle \rightarrow\left\langle z^{*}-w^{*}, z^{* *}\right\rangle$ and $\left\|s_{n}^{*}-z^{*}\right\| \rightarrow 0$ as $n \rightarrow \infty$.

(d) For all $\left(w, w^{*}\right) \in E \times E^{*}$, there exists a sequence $\left\{\left(s_{n}, s_{n}^{*}\right)\right\}_{n \geq 1}$ of elements of $G(S)$ such that

$$
\left\langle s_{n}-w, s_{n}^{*}-w^{*}\right\rangle \rightarrow\left\langle z^{*}-w^{*}, z^{* *}-\widehat{w}\right\rangle \text { and }\left\|s_{n}^{*}-z^{*}\right\| \rightarrow 0 \text { as } n \rightarrow \infty .
$$

Proof. It is immediate from the argument already used in Lemma 7.4 that (a) $\Longrightarrow(\mathrm{b})$.

Now suppose that (b) is true and $\left(w, w^{*}\right) \in E \times E^{*}$. Let $T:=S-w^{*}$. Clearly, $T$ is closed monotone and quasidense.

By direct computation, $\varphi_{S}^{* *}\left(z^{* *}, \widehat{z^{*}}\right)=\varphi_{T}^{* *}\left(z^{* *}, \widehat{z^{*}}-\widehat{w^{*}}\right)+\left\langle w^{*}, z^{* *}\right\rangle$. From (b), $\varphi_{T}^{* *}\left(z^{* *}, \widehat{z^{*}}-\widehat{w^{*}}\right)=\left\langle z^{*}-w^{*}, z^{* *}\right\rangle$. From Lemma 17.4((c) $\left.\Longrightarrow(\mathrm{d})\right)$, there exists a sequence $\left\{\left(t_{n}, t_{n}^{*}\right)\right\}_{n \geq 1}$ of elements of $G(T)$ such that $\lim _{n}\left\langle t_{n}, t_{n}^{*}\right\rangle=\left\langle z^{*}-w^{*}, z^{* *}\right\rangle$ and $\lim _{n}\left\|t_{n}^{*}-\left(z^{*}-w^{*}\right)\right\|=0$. Let $\left(s_{n}, s_{n}^{*}\right):=\left(t_{n}, t_{n}^{*}+w^{*}\right) \in G(S)$. Then $\lim _{n}\left\langle s_{n}, s_{n}^{*}-w^{*}\right\rangle=\left\langle z^{*}-w^{*}, z^{* *}\right\rangle$ and $\lim _{n}\left\|s_{n}^{*}-z^{*}\right\|=0$, giving (c). 
Now suppose that (c) is true. Then, for all $\left(w, w^{*}\right) \in E \times E^{*}$,

$$
\begin{aligned}
\left\langle s_{n}-w, s_{n}^{*}-w^{*}\right\rangle & -\left\langle z^{*}-w^{*}, z^{* *}-\widehat{w}\right\rangle \\
& =\left\langle s_{n}, s_{n}^{*}-w^{*}\right\rangle-\left\langle w, s_{n}^{*}-z^{*}\right\rangle+\left\langle w^{*}-z^{*}, z^{* *}\right\rangle \\
& \rightarrow\left\langle z^{*}-w^{*}, z^{* *}\right\rangle+0+\left\langle w^{*}-z^{*}, z^{* *}\right\rangle=0 .
\end{aligned}
$$

Thus (7.8) is satisfied, and so (d) is true.

Suppose, finally, that (d) is true. Then, reversing the argument above,

$$
\left\langle s_{n}, s_{n}^{*}-w^{*}\right\rangle-\left\langle w, s_{n}^{*}-z^{*}\right\rangle+\left\langle w^{*}-z^{*}, z^{* *}\right\rangle \rightarrow 0,
$$

from which

$$
\begin{aligned}
\left\langle w, z^{*}\right\rangle+\left\langle w^{*}, z^{* *}\right\rangle & -\left\langle z^{*}, z^{* *}\right\rangle=\lim _{n}\left[\left\langle s_{n}, w^{*}\right\rangle+\left\langle w, s_{n}^{*}\right\rangle-\left\langle s_{n}, s_{n}^{*}\right\rangle\right] \\
& \leq \sup _{\left(s, s^{*}\right) \in G(S)}\left[\left\langle s, w^{*}\right\rangle+\left\langle w, s^{*}\right\rangle-\left\langle s, s^{*}\right\rangle\right]=\varphi_{S}\left(w, w^{*}\right) .
\end{aligned}
$$

Consequently, $\left\langle w, z^{*}\right\rangle+\left\langle w^{*}, z^{* *}\right\rangle-\varphi_{S}\left(w, w^{*}\right) \leq\left\langle z^{*}, z^{* *}\right\rangle$. Taking the supremum over $\left(w, w^{*}\right), \varphi_{S}^{*}\left(z^{*}, z^{* *}\right) \leq\left\langle z^{*}, z^{* *}\right\rangle$, and it follows from Theorem 2.7 and (3.1) that (a) is true.

Remark 7.6. The equivalence of (a) and (b) above was established in [23, Lemma 12.4(a), p. 1047].

\section{Type (FP)}

Lemma 8.1 below will simplify the computations in Theorem 8.3 considerably:

Lemma 8.1. Let $T: E \rightrightarrows E^{*}$ be closed, monotone and quasidense, $\widetilde{V}$ be an open convex subset of $E^{*}, \widetilde{V} \ni 0, \widetilde{V} \cap R(T) \neq \emptyset$ and

$$
\left(t, t^{*}\right) \in G(T) \text { and } t^{*} \in \widetilde{V} \quad \Longrightarrow \quad\left\langle t, t^{*}\right\rangle \geq 0 .
$$

Then $(0,0) \in G(T)$.

Proof. Let $y^{*} \in \widetilde{V} \cap R(T)$. Since the segment $\left[0, y^{*}\right]$ is a compact subset of the open set $\widetilde{V}$, we can choose $\varepsilon>0$ so that $\widetilde{K}:=\left[0, y^{*}\right]+\varepsilon E_{1}^{*} \subset \widetilde{V}$. From (2.7), $R(T) \cap \operatorname{int} R\left(\partial \tau_{\widetilde{K}}\right)=R(T) \cap \operatorname{int} \widetilde{K} \ni y^{*}$. We now define the multifunction $P: E \rightrightarrows E^{*}$ by $P(y):=\left(T^{\mathbb{F}}+\partial \tau_{\widetilde{K}^{\mathbb{F}}}\right)^{-1}(\widehat{y})$. (2.5) and Theorem 4.2 imply that $P$ is closed and quasidense. Let $\eta>0$. Then there exists $\left(y, z^{*}\right) \in G(P)$ such that

$$
\frac{1}{2}\|y\|^{2}+\frac{1}{2}\left\|z^{*}\right\|^{2}+\left\langle y, z^{*}\right\rangle<\eta .
$$

We can choose $z^{* *} \in T^{\mathbb{F}}\left(z^{*}\right)$ such that $\widehat{y}-z^{* *} \in \partial \tau_{\widetilde{K}}{ }^{\mathbb{F}}\left(z^{*}\right)$. From Lemma 3.4 $z^{*} \in \widetilde{K} \subset \widetilde{V}$ and $\left\langle z^{*}, \widehat{y}-z^{* *}\right\rangle=\sup \left\langle\widetilde{K}, \widehat{y}-z^{* *}\right\rangle \geq \varepsilon\left\|\widehat{y}-z^{* *}\right\| \geq 0$. Thus

$$
\left\langle y, z^{*}\right\rangle=\left\langle z^{*}, \widehat{y}\right\rangle \geq \varepsilon\left\|\widehat{y}-z^{* *}\right\|+\left\langle z^{*}, z^{* *}\right\rangle \geq\left\langle z^{*}, z^{* *}\right\rangle .
$$


From Lemma 7.4 there exists a sequence $\left\{\left(t_{n}, t_{n}^{*}\right)\right\}_{n \geq 1}$ of elements of $G(T)$ such that $\left\langle t_{n}, t_{n}^{*}\right\rangle \rightarrow\left\langle z^{*}, z^{* *}\right\rangle$ and $\left\|t_{n}^{*}-z^{*}\right\| \rightarrow 0$ as $n \rightarrow \infty$. If $n$ is sufficiently large, $t_{n}^{*} \in \widetilde{V}$, and so, from (8.1), $\left\langle t_{n}, t_{n}^{*}\right\rangle \geq 0$. Passing to the limit, $\left\langle z^{*}, z^{* *}\right\rangle \geq 0$. Combining this with (8.2) and (8.3),

$$
\frac{1}{2}\|y\|^{2}+\frac{1}{2}\left\|z^{*}\right\|^{2}<\eta \text {. }
$$

Taking $\eta$ arbitrarily small and using the fact that $P$ is closed, we derive that $(0,0) \in G(P)$. Repeating the argument already used above, we can choose $z_{0}^{* *} \in$ $T^{\mathbb{F}}(0)$ such that $\langle 0,0\rangle \geq \varepsilon\left\|0-z_{0}^{* *}\right\|$. Thus $z_{0}^{* *}=0$, from which $(0,0)=\left(0, z_{0}^{* *}\right) \in G\left(T^{\mathbb{F}}\right)$, and so (3.2) implies that $(0,0) \in G(T)$.

Definition 8.2. Let $S: E \rightrightarrows E^{*}$ be monotone. We say that $S$ is of type (FP) or locally maximally monotone if whenever $\widetilde{U}$ is a convex open subset of $E^{*}$, $\widetilde{U} \cap R(S) \neq \emptyset,\left(w, w^{*}\right) \in E \times \widetilde{U}$ and

$$
\left(s, s^{*}\right) \in G(S) \text { and } s^{*} \in \widetilde{U} \quad \Longrightarrow \quad\left\langle s-w, s^{*}-w^{*}\right\rangle \geq 0,
$$

then $\left(w, w^{*}\right) \in G(S)$. (If we take $\widetilde{U}=E^{*}$, we see that every monotone multifunction of type (FP) is maximally monotone.)

Theorem 8.3 (The type (FP) criterion for quasidensity). Let $S: E \rightrightarrows E^{*}$ be maximally monotone. Then the conditions (a), (b) and (c) are equivalent.

(a) $S$ is quasidense.

(b) $S$ is of type (FP).

(c) For all $\left(w^{*}, w^{* *}\right) \in E^{*} \times E^{* *}, \inf _{\left(s, s^{*}\right) \in G(S)}\left\langle s^{*}-w^{*}, \widehat{s}-w^{* *}\right\rangle \leq 0$.

Proof. (a) $\Longrightarrow\left(\right.$ b). Let $\widetilde{U}$ be an open convex subset of $E^{*}, \widetilde{U} \cap R(S) \neq \emptyset$, $\left(w, w^{*}\right) \in E \times \widetilde{U}$ and (8.4) be satisfied. It follows easily from Lemma 8.1 with $T$ defined so that $G(T)=G(S)-\left(w, w^{*}\right)$ and $\widetilde{V}:=\widetilde{U}-w^{*}$ that $\left(w, w^{*}\right) \in G(S)$.

(b) $\Longrightarrow(\mathrm{c})$. Let $S$ be of type $(\mathrm{FP})$ and $\inf _{\left(s, s^{*}\right) \in G(S)}\left\langle s^{*}-w^{*}, \widehat{s}-w^{* *}\right\rangle>0$. We choose $\varepsilon>0$ so that $\inf _{\left(s, s^{*}\right) \in G(S)}\left\langle s^{*}-w^{*}, \widehat{s}-w^{* *}\right\rangle>\varepsilon$ and define $\eta:=\varepsilon /\left(2\left\|w^{* *}\right\|+2\right)$. Let $y^{*} \in R(T)$. From Lemma 7.1 with $X=E$, $f_{0}:=\|\cdot\|-\left\|w^{* *}\right\|$ and $f_{1}:=y^{*}-\left\langle y^{*}, w^{* *}\right\rangle\left(\right.$ so that $\left.f_{0}{ }^{* *}\left(w^{* *}\right)=f_{1}{ }^{* *}\left(w^{* *}\right)=0\right)$ there exists $w \in E$ such that

$$
\|w\| \leq\left\|w^{* *}\right\|+1 \text { and }\left\langle y^{*}, \widehat{w}-w^{* *}\right\rangle \leq \eta .
$$

Let $T: E \rightrightarrows E^{*}$ be defined so that $G(T)=G(S)-\left(w, w^{*}\right)$. Then we have

$$
\left(t, t^{*}\right) \in G(T) \quad \Longrightarrow \quad\left\langle t^{*}, \widehat{t}+\widehat{w}-w^{* *}\right\rangle>\varepsilon
$$

Let $\widetilde{U}:=\left[0, y^{*}\right]+\left\{z^{*} \in E^{*}:\left\|z^{*}\right\|<\eta\right\} . \widetilde{U}$ is convex and open and $\widetilde{U} \cap R(T) \neq \emptyset$. We now prove that

$$
\left(t, t^{*}\right) \in G(T) \text { and } t^{*} \in \widetilde{U} \quad \Longrightarrow \quad\left\langle t, t^{*}\right\rangle \geq 0 .
$$


To this end, let $\left(t, t^{*}\right) \in G(T)$ and $t^{*} \in \widetilde{U}$. Then there exists $\lambda \in[0,1]$ such that $\left\|t^{*}-\lambda y^{*}\right\|<\eta$. Combining this with (8.5) and (8.6),

$$
\begin{aligned}
\left\langle t, t^{*}\right\rangle & =\left\langle t^{*}, \widehat{t}+\widehat{w}-w^{* *}\right\rangle-\left\langle t^{*}, \widehat{w}-w^{* *}\right\rangle>\varepsilon-\left\langle t^{*}, \widehat{w}-w^{* *}\right\rangle \\
& =\varepsilon-\left\langle t^{*}-\lambda y^{*}, \widehat{w}-w^{* *}\right\rangle-\lambda\left\langle y^{*}, \widehat{w}-w^{* *}\right\rangle \geq \varepsilon-\left\|\widehat{w}-w^{* *}\right\| \eta-\lambda \eta \\
& \geq \varepsilon-\left(2\left\|w^{* *}\right\|+1\right) \eta-\lambda \eta \geq \varepsilon-\left(2\left\|w^{* *}\right\|+2\right) \eta=0 .
\end{aligned}
$$

This completes the proof of (8.7). Clearly, $T$ is of type (FP) and so, from Definition 8.2 $(0,0) \in G(T)$. But then (8.6) would give $\left\langle 0, \widehat{w}-w^{* *}\right\rangle>\varepsilon$, which is impossible.

$(\mathrm{c}) \Longrightarrow(\mathrm{a})$. From Lemma 2.5, for all $\left(w^{*}, w^{* *}\right) \in E^{*} \times E^{* *}$,

$$
\begin{aligned}
\inf _{\left(s, s^{*}\right) \in G(S)}\langle & \left.s^{*}-w^{*}, \widehat{s}-w^{* *}\right\rangle \\
& =\left\langle w^{*}, w^{* *}\right\rangle+\inf _{\left(s, s^{*}\right) \in G(S)}\left[\left\langle s, s^{*}\right\rangle-\left\langle s, w^{*}\right\rangle-\left\langle s^{*}, w^{* *}\right\rangle\right] \\
& =\left\langle w^{*}, w^{* *}\right\rangle+\inf _{\left(s, s^{*}\right) \in G(S)}\left[\varphi_{S}\left(s, s^{*}\right)-\left\langle s, w^{*}\right\rangle-\left\langle s^{*}, w^{* *}\right\rangle\right] \\
& \geq\left\langle w^{*}, w^{* *}\right\rangle+\inf _{\left(x, x^{*}\right) \in E \times E^{*}}\left[\varphi_{S}\left(x, x^{*}\right)-\left\langle x, w^{*}\right\rangle-\left\langle x^{*}, w^{* *}\right\rangle\right] \\
& =\left\langle w^{*}, w^{* *}\right\rangle-\varphi_{S}\left(w^{*}, w^{* *}\right) .
\end{aligned}
$$

Thus (c) implies that, for all $\left(w^{*}, w^{* *}\right) \in E^{*} \times E^{* *}, \varphi_{S}{ }^{*}\left(w^{*}, w^{* *}\right) \geq\left\langle w^{*}, w^{* *}\right\rangle$, and it follows from Theorem 2.7 that $S$ is quasidense.

Remark 8.4. Condition (c) above is exactly that $S$ is of type (NI). So the fact that $(\mathrm{a}) \Longleftrightarrow(\mathrm{c})$ above can easily be deduced from the results proved by Marques Alves and Svaiter in [11, Theorem 1.2(1 27. Theorem 4.1, pp. 1027-1028]. Of course the conditions contained in [11, Theorem 1.2 (3 and 4)] are closely related to our definition of quasidensity. See [23, Theorem 6.10, p. 1031] for a more general result. The implication (b) $\Longrightarrow(\mathrm{c})$ above was established by Bauschke, Borwein, Wang and Yao in [2, Theorem 3.1, pp. 1878-1879]. It would be nice to find a proof of $(\mathrm{a}) \Longrightarrow(\mathrm{b})$ above free of the complexities of Section 7 , but this seems a hard problem.

So the equivalences outlined in Theorem 8.3 are already in the literature, but the approach outlined in this paper shows that Theorems 6.1 and 6.2 give additional information about these classes of maximal monotone multifunctions and the equivalent classes "type (D)", "dense type" and "type (ED)", as well as those that satisfy the "negative alignment criterion" of [23, Theorem 11.6, p. 1045].

\section{Appendix 1}

In Appendix 1, we discuss the function $\theta_{S}$ briefly, show the connection with the Gossez extension, and give a self-contained proof of Theorem 3.6.

Let $S$ be closed, monotone and quasidense. We define the function $\left.\left.\theta_{S}: E^{*} \times E^{* *} \rightarrow\right]-\infty, \infty\right]$ by

$$
\theta_{S}\left(w^{*}, w^{* *}\right):=\sup _{\left(s, s^{*}\right) \in G(S)}\left[\left\langle s, w^{*}\right\rangle+\left\langle s^{*}, w^{* *}\right\rangle-\left\langle s, s^{*}\right\rangle\right] .
$$


Then condition Theorem 8.3(c) can be put in the equivalent form:

$$
\text { for all }\left(w^{*}, w^{* *}\right) \in E^{*} \times E^{* *}, \theta_{S}\left(w^{*}, w^{* *}\right) \geq\left\langle w^{*}, w^{* *}\right\rangle .
$$

Now let $\left(w^{*}, w^{* *}\right) \in E^{*} \times E^{* *}$. An examination of the proof that $(\mathrm{c}) \Longrightarrow$ (a) in Theorem 8.3 shows that $\varphi_{S}{ }^{*}\left(w^{*}, w^{* *}\right) \geq \theta_{S}\left(w^{*}, w^{* *}\right)$. See [23, Eq. (21), p. 1029]. We also have

$$
\begin{aligned}
\theta_{S}{ }^{*}\left(w^{* *}, \widehat{w^{*}}\right) & =\sup _{\left(x^{*}, x^{* *}\right) \in E^{*} \times E^{* *}}\left[\left\langle x^{*}, w^{* *}\right\rangle+\left\langle w^{*}, x^{* *}\right\rangle-\theta_{S}\left(x^{*}, x^{* *}\right)\right] \\
& \geq \sup _{\left(x, x^{*}\right) \in E \times E^{*}}\left[\left\langle x^{*}, w^{* *}\right\rangle+\left\langle x, w^{*}\right\rangle-\theta_{S}\left(x^{*}, \widehat{x}\right)\right] .
\end{aligned}
$$

Since $\theta_{S}\left(x^{*}, \widehat{x}\right)=\varphi_{S}\left(x, x^{*}\right)$, it follows that $\theta_{S}{ }^{*}\left(w^{* *}, \widehat{w^{*}}\right) \geq \varphi_{S}{ }^{*}\left(w^{*}, w^{* *}\right)$. Consequently, using (9.1),

$$
\theta_{S}{ }^{*}\left(w^{* *}, \widehat{w^{*}}\right) \geq \varphi_{S}{ }^{*}\left(w^{*}, w^{* *}\right) \geq \theta_{S}\left(w^{*}, w^{* *}\right) \geq\left\langle w^{*}, w^{* *}\right\rangle .
$$

From Lemma 7.3 . $\theta_{S}\left(z^{*}, z^{* *}\right)=\left\langle z^{*}, z^{* *}\right\rangle$ implies that $\theta_{S}{ }^{*}\left(z^{* *}, \widehat{z^{*}}\right)=\left\langle z^{*}, z^{* *}\right\rangle$. Combining this with (9.2) and (3.1), we see that

$$
\begin{aligned}
G\left(S^{\mathbb{F}}\right): & =\left\{\left(w^{*}, w^{* *}\right): \varphi_{S}{ }^{*}\left(w^{*}, w^{* *}\right)=\left\langle w^{*}, w^{* *}\right\rangle\right\} \\
& =\left\{\left(w^{*}, w^{* *}\right): \theta_{S}\left(w^{*}, w^{* *}\right)=\left\langle w^{*}, w^{* *}\right\rangle\right\} \\
& =\left\{\left(w^{*}, w^{* *}\right): \theta_{S}{ }^{*}\left(w^{* *}, \widehat{w^{*}}\right)=\left\langle w^{*}, w^{* *}\right\rangle\right\} .
\end{aligned}
$$

In particular, using the definition of $\theta_{S},\left(y^{*}, y^{* *}\right) \in G\left(S^{\mathbb{F}}\right)$ exactly when $\left(y^{* *}, y^{*}\right)$ is in the Gossez extension of $G(S)$ (see [8, Lemma 2.1,p. 275]).

Finally, we show how (9.3) leads to a proof of Theorem 3.6. To this end, let $\left(w^{*}, w^{* *}\right) \in E^{*} \times E^{* *}$ and $\inf _{\left(z^{*}, z^{* *}\right) \in G\left(S^{\mathbb{P}}\right)}\left\langle z^{*}-w^{*}, z^{* *}-w^{* *}\right\rangle \geq 0$. From (3.2), $\inf _{\left(t, t^{*}\right) \in G(S)}\left\langle t^{*}-w^{*}, \widehat{t}-w^{* *}\right\rangle \geq 0$. It follows from Theorem 8.3 ((a) $\left.\Longrightarrow(\mathrm{c})\right)$ that $\inf _{\left(t, t^{*}\right) \in G(S)}\left\langle t^{*}-w^{*}, \widehat{t}-w^{* *}\right\rangle=0$, and (9.3) now implies that $\left(w^{*}, w^{* *}\right) \in G\left(S^{\mathbb{F}}\right)$.

\section{Appendix 2}

In Appendix 2, we give a proof of Lemma 8.1 that does not use Theorem 4.2 but uses instead Rockafellar's formula for the conjugate of a sum and version of the Fenchel duality theorem.

Lemma 10.1. Let $T: E \rightrightarrows E^{*}$ be closed, monotone and quasidense and $\widetilde{K}$ be a $w\left(E^{*}, E\right)$-compact convex subset of $E^{*}$ such that $R(T) \cap \operatorname{int} \widetilde{K} \neq \emptyset$. Then there exist $z^{*} \in \widetilde{K}$ and $z^{* *}, x^{* *} \in E^{* *}$ such that

$$
\varphi_{T}^{*}\left(z^{*}, z^{* *}\right)+\sup \left\langle\widetilde{K}, x^{* *}-z^{* *}\right\rangle+\frac{1}{2}\left\|\left(z^{*}, x^{* *}\right)\right\|^{2} \leq 0,
$$

and

$$
z^{* *} \in T^{\mathbb{F}}\left(z^{*}\right) \text { and }\left\langle z^{*}, z^{* *}\right\rangle+\sup \left\langle\widetilde{K}, x^{* *}-z^{* *}\right\rangle+\frac{1}{2}\left\|\left(z^{*}, x^{* *}\right)\right\|^{2} \leq 0 .
$$


Proof. For all $\left(x, x^{*}\right) \in E \times E^{*}$, let $h\left(x, x^{*}\right):=\mathbb{I}_{\widetilde{K}}\left(x^{*}\right)$. Clearly, dom $h=E \times \widetilde{K}$, and so $h \in \mathcal{P C} \mathcal{L S C}\left(E \times E^{*}\right)$. Also, for all $\left(y^{*}, y^{* *}\right) \in E^{*} \times E^{* *}$,

$$
h^{*}\left(y^{*}, y^{* *}\right)=\mathbb{I}_{\{0\}}\left(y^{*}\right)+\sup \left\langle\widetilde{K}, y^{* *}\right\rangle .
$$

If $\left(t, t^{*}\right) \in G(T)$ and $t^{*} \in \operatorname{int} \widetilde{K}$ then, from Lemma 2.5. $\varphi_{T}\left(t, t^{*}\right)=\left\langle t, t^{*}\right\rangle \in \mathbb{R}$ and so $\operatorname{dom} \varphi_{T} \cap \operatorname{int} \operatorname{dom} h=\operatorname{dom} \varphi_{T} \cap(E \times \operatorname{int} \widetilde{K}) \neq \emptyset$. Thus, from Rockafellar's formula for the conjugate of a sum, [15, Theorem 3(a), pp. 85-86], and (10.3), for all $\left(z^{*}, x^{* *}\right) \in E^{*} \times E^{* *}$,

$$
\begin{aligned}
& \sup _{\left(y, x^{*}\right) \in E \times \widetilde{K}}\left[\left\langle y, z^{*}\right\rangle+\left\langle x^{*}, x^{* *}\right\rangle-\varphi_{T}\left(y, x^{*}\right)\right] \\
& =\sup _{\left(y, x^{*}\right) \in E \times E^{*}}\left[\left\langle y, z^{*}\right\rangle+\left\langle x^{*}, x^{* *}\right\rangle-\left(\varphi_{T}+h\right)\left(y, x^{*}\right)\right] \\
& =\left(\varphi_{T}+h\right)^{*}\left(z^{*}, x^{* *}\right) . \\
& =\min _{\left(w^{*}, z^{* *}\right) \in E^{*} \times E^{* *}}\left[\varphi_{T}^{*}\left(w^{*}, z^{* *}\right)+h^{*}\left(z^{*}-w^{*}, x^{* *}-z^{* *}\right)\right] \\
& =\min _{\left(w^{*}, z^{* *}\right) \in E^{*} \times E^{* *}}\left[\varphi_{T}{ }^{*}\left(w^{*}, z^{* *}\right)+\mathbb{I}_{\{0\}}\left(z^{*}-w^{*}\right)+\sup \left\langle\widetilde{K}, x^{* *}-z^{* *}\right\rangle\right] \\
& =\min _{z^{* *} \in E^{* *}}\left[\varphi_{T}^{*}\left(z^{*}, z^{* *}\right)+\sup \left\langle\widetilde{K}, x^{* *}-z^{* *}\right\rangle\right] .
\end{aligned}
$$

For all $\left(x, x^{*}\right) \in E \times E^{*}$, let

$$
f\left(x, x^{*}\right):= \begin{cases}\inf _{y \in E}\left[\varphi_{T}\left(y, x^{*}\right)+\max \langle x-y, \widetilde{K}\rangle\right] & \left(x^{*} \in \widetilde{K}\right) ; \\ \infty & \left(x^{*} \notin \widetilde{K}\right) .\end{cases}
$$

Since $\widetilde{K}$ is a $w\left(E^{*}, E\right)$-closed convex subset of $E^{*}$, we have

$$
\sup _{w \in E}\left[\left\langle w, z^{*}\right\rangle-\max \langle w, \widetilde{K}\rangle\right]=\mathbb{I}_{\widetilde{K}}\left(z^{*}\right)
$$

If $x^{*} \in \widetilde{K}$ then, from Lemma 2.5. for all $y \in E, \varphi_{T}\left(y, x^{*}\right)+\max \langle x-y, \widetilde{K}\rangle \geq$ $\left\langle y, x^{*}\right\rangle+\left\langle x-y, x^{*}\right\rangle=\left\langle x, x^{*}\right\rangle$ thus,

$$
\text { for all }\left(x, x^{*}\right) \in E \times E^{*}, \quad f\left(x, x^{*}\right) \geq\left\langle x, x^{*}\right\rangle .
$$

Thus $\left.\left.f: E \times E^{*} \rightarrow\right]-\infty, \infty\right]$ and $f$ is easily seen to be convex. On the other hand, if $\left(t, t^{*}\right) \in G(T)$ and $t^{*} \in \widetilde{K}$ then (taking $y=t$ ),

$$
f\left(t, t^{*}\right) \leq \varphi_{T}\left(t, t^{*}\right)+\max \langle t-t, \widetilde{K}\rangle=\left\langle t, t^{*}\right\rangle+0=\left\langle t, t^{*}\right\rangle .
$$

Thus $f$ is proper. From (10.4) and (10.5),

$$
\begin{aligned}
& f^{*}\left(z^{*}, x^{* *}\right) \\
& \quad=\sup _{\left(x, x^{*}\right) \in E \times \widetilde{K}, y \in E}\left[\left\langle x, z^{*}\right\rangle+\left\langle x^{*}, x^{* *}\right\rangle-\varphi_{T}\left(y, x^{*}\right)-\max \langle x-y, \widetilde{K}\rangle\right] \\
& \quad=\sup _{\left(w, x^{*}\right) \in E \times \widetilde{K}, y \in E}\left[\left\langle w+y, z^{*}\right\rangle+\left\langle x^{*}, x^{* *}\right\rangle-\varphi_{T}\left(y, x^{*}\right)-\max \langle w, \widetilde{K}\rangle\right] \\
& \quad=\sup _{\left(y, x^{*}\right) \in E \times \widetilde{K}, w \in E}\left[\left\langle y, z^{*}\right\rangle+\left\langle x^{*}, x^{* *}\right\rangle-\varphi_{T}\left(y, x^{*}\right)+\left\langle w, z^{*}\right\rangle-\max \langle w, \widetilde{K}\rangle\right] \\
& \quad=\min _{z^{* *} \in E^{* *}}\left[\varphi_{T}^{*}\left(z^{*}, z^{* *}\right)+\sup \left\langle\widetilde{K}, x^{* *}-z^{* *}\right\rangle\right]+\mathbb{I}_{\widetilde{K}}\left(z^{*}\right) .
\end{aligned}
$$


From (10.6),

for all $\left(x, x^{*}\right) \in E \times E^{*}, \quad f\left(x, x^{*}\right)+\frac{1}{2}\left\|\left(x, x^{*}\right)\right\|^{2} \geq\left\langle x, x^{*}\right\rangle+\frac{1}{2}\left\|\left(x, x^{*}\right)\right\|^{2} \geq 0$.

Rockafellar's version of the Fenchel duality theorem, [15, Theorem 3(a), p. 85], gives $\left(z^{*}, x^{* *}\right) \in E^{*} \times E^{* *}$ such that $f^{*}\left(z^{*}, x^{* *}\right)+\frac{1}{2}\left\|-\left(z^{*}, x^{* *}\right)\right\|^{2} \leq 0$. From (10.7), $z^{*} \in \widetilde{K}$ and there exists $z^{* *} \in E^{* *}$ such that (10.1) is satisfied. It follows from this that $\varphi_{T}{ }^{*}\left(z^{*}, z^{* *}\right)+\left\langle z^{*}, x^{* *}-z^{* *}\right\rangle+\frac{1}{2}\left\|\left(z^{*}, x^{* *}\right)\right\|^{2} \leq 0$. Since $\left\langle z^{*}, x^{* *}\right\rangle+\frac{1}{2}\left\|\left(z^{*}, x^{* *}\right)\right\|^{2} \geq 0$, this implies that $\varphi_{T}^{*}\left(z^{*}, z^{* *}\right)-\left\langle z^{*}, z^{* *}\right\rangle \leq 0$. From Theorem 2.7 and (3.1), $\varphi_{T}^{*}\left(z^{*}, z^{* *}\right)=\left\langle z^{*}, z^{* *}\right\rangle$ and $z^{* *} \in T^{\mathbb{F}}\left(z^{*}\right)$ and so (10.2) follows from (10.1).

Here is the promised proof of Lemma 8.1

Lemma 10.2. Let $T: E \rightrightarrows E^{*}$ be closed, monotone and quasidense, $\widetilde{V}$ be an open convex subset of $E^{*}, \widetilde{V} \ni 0, \widetilde{V} \cap R(T) \neq \emptyset$ and

$$
\left(t, t^{*}\right) \in G(T) \text { and } t^{*} \in \widetilde{V} \quad \Longrightarrow \quad\left\langle t, t^{*}\right\rangle \geq 0 .
$$

Then $(0,0) \in G(T)$.

Proof. Let $y^{*} \in \widetilde{V} \cap R(T)$. Since the segment $\left[0, y^{*}\right]$ is a compact subset of the open set $\widetilde{V}$, we can choose $\varepsilon>0$ so that $\widetilde{K}:=\left[0, y^{*}\right]+\varepsilon E_{1}^{*} \subset \widetilde{V}$. From (2.7), $R(T) \cap \operatorname{int} R\left(\partial \tau_{\widetilde{K}}\right)=R(T) \cap \operatorname{int} \widetilde{K} \ni y^{*}$. Using Lemma 10.1, there exist $z^{*} \in \widetilde{K}$ and $z^{* *}, x^{* *} \in E^{* *}$ satisfying (10.2).

From Lemma 7.4, there exists a sequence $\left\{\left(t_{n}, t_{n}^{*}\right)\right\}_{n \geq 1}$ of elements of $G(T)$ such that $\left\langle t_{n}, t_{n}^{*}\right\rangle \rightarrow\left\langle z^{*}, z^{* *}\right\rangle$ and $\left\|t_{n}^{*}-z^{*}\right\| \rightarrow 0$ as $n \rightarrow \infty$. If $n$ is sufficiently large, $t_{n}^{*} \in \widetilde{V}$, and so, from (10.8), $\left\langle t_{n}, t_{n}^{*}\right\rangle \geq 0$. Passing to the limit, $\left\langle z^{*}, z^{* *}\right\rangle \geq 0$, and so (10.2) now implies that

$$
\sup \left\langle\widetilde{K}, x^{* *}-z^{* *}\right\rangle+\frac{1}{2}\left\|\left(z^{*}, x^{* *}\right)\right\|^{2} \leq 0 .
$$

Since $\sup \left\langle\widetilde{K}, x^{* *}-z^{* *}\right\rangle \geq \varepsilon\left\|x^{* *}-z^{* *}\right\|, \quad$ it follows that $z^{* *}=x^{* *}, z^{*}=0$ and $x^{* *}=0$. Consequently, $0=z^{* *} \in T^{\mathbb{F}}\left(z^{*}\right)=T^{\mathbb{F}}(0)$, and (3.2) implies that $(0,0) \in G(T)$.

Remark 10.3. In the context of Lemma 10.1, one can in fact prove that $\left\langle z^{*}, x^{* *}\right\rangle+\frac{1}{2}\left\|\left(z^{*}, x^{* *}\right)\right\|^{2}=0$ and $\left\langle z^{*}, x^{* *}-z^{* *}\right\rangle=\sup \left\langle\widetilde{K}, x^{* *}-z^{* *}\right\rangle$.

We now sketch the "dual" version of Lemma 10.1. Lemma 10.4, which can be used to prove Theorem 5.2 however this proof of Theorem 5.2 does require Lemma 7.4 and the appearance of $\ddot{K}$ makes Lemma 10.4 innately more "technical" than Lemma 10.1

Lemma 10.4. Let $T: E \rightrightarrows E^{*}$ be closed, monotone and quasidense and $K$ be a bounded closed convex subset of $E$ such that $D(T) \cap \operatorname{int} K \neq \emptyset$. Let $\ddot{K}$ be the $w\left(E^{* *}, E^{*}\right)$-closure of $\widehat{K}$ in $E^{* *}$. Then there exist $z^{* *} \in \ddot{K}$ and $z^{*}, v^{*} \in E^{*}$ such that

$$
z^{* *} \in T^{\mathbb{F}}\left(z^{*}\right) \text { and }\left\langle z^{*}, z^{* *}\right\rangle+\sup \left\langle K, v^{*}-z^{*}\right\rangle+\frac{1}{2}\left\|\left(v^{*}, z^{* *}\right)\right\|^{2} \leq 0 .
$$


Proof. For all $\left(x, x^{*}\right) \in E \times E^{*}$, let

$$
f\left(x, x^{*}\right):= \begin{cases}\inf _{y^{*} \in E^{*}}\left[\varphi_{T}\left(x, y^{*}\right)+\sup \left\langle K, x^{*}-y^{*}\right\rangle\right] & (x \in K) ; \\ \infty & (x \notin K) .\end{cases}
$$

Then $f$ is proper and convex, for all $\left(v^{*}, z^{* *}\right) \in E^{*} \times E^{* *}$,

$$
f^{*}\left(v^{*}, z^{* *}\right)=\min _{z^{*} \in E^{*}}\left[\varphi_{T}^{*}\left(z^{*}, z^{* *}\right)+\sup \left\langle K, v^{*}-z^{*}\right\rangle\right]+I_{\ddot{K}}\left(z^{* *}\right)
$$

and, for all $\left(x, x^{*}\right) \in E \times E^{*}, \quad f\left(x, x^{*}\right)+\frac{1}{2}\left\|\left(x, x^{*}\right)\right\|^{2} \geq 0$. The result now

follows from Rockafellar's version of the Fenchel duality theorem, just as in Lemma 10.1

\section{References}

[1] H. H. Bauschke and S. Simons, Stronger maximal monotonicity properties of linear operators, Bull. Austral. Math. Soc. 60 (1999), 163-174.

[2] H. Bauschke, J. M. Borwein, X. Wang and L. Yao, Every maximally monotone operator of Fitzpatrick-Phelps type is actually of dense type, Optim. Lett. 6 (2012), 1875-1881. DOI: 10.1007/s11590-011-0383-2

[3] A. Brøndsted and R.T. Rockafellar, On the subdifferentiability of convex functions, Proc. Amer. Math. Soc. 16 (1965), 605-611.

[4] S. Fitzpatrick, Representing monotone operators by convex functions, Workshop/ Miniconference on Functional Analysis and Optimization (Canberra, 1988), 59-65, Proc. Centre Math. Anal. Austral. Nat. Univ., 20, Austral. Nat. Univ., Canberra, 1988.

[5] S. P. Fitzpatrick and R. R. Phelps, Bounded approximants to monotone operators on Banach spaces, Ann. Inst. Henri Poincaré, Analyse non linéaire 9 (1992), 573-595.

[6] S. P. Fitzpatrick and R. R. Phelps, Some properties of maximal monotone operators on nonreflexive Banach spaces, Set-Valued Analysis 3(1995), 5169.

[7] S. P. Fitzpatrick and S. Simons, On the pointwise maximum of convex functions, Proc. Amer. Math. Soc. 128 (2000), 3553-3561.

[8] J.-P. Gossez, Opérateurs monotones non linéaires dans les espaces de Banach non réflexifs, J. Math. Anal. Appl. 34 (1971), 371-395.

[9] N. Krylov, Properties of monotone mappings, Lith. Math. J. 22 (1982), $140-145$.

[10] M. Marques Alves and B. F. Svaiter, A new old class of maximal monotone operators, J. of Convex Anal., 16(2009), 881-890. 
[11] M. Marques Alves and B. F. Svaiter, On the surjectivity properties of perturbations of maximal monotone operators in non-reflexive Banach spaces, J. of Convex Anal., 18(2011), 209-226.

[12] J.-E. Martínez-Legaz and M. Théra, A convex representation of maximal monotone operators, J. Nonlinear Convex Anal. 2 (2001), 243-247.

[13] J.--J. Moreau, Fonctionelles convexes, Séminaire sur les équations aux derivées partielles, Lecture notes, Collège de France, Paris 1966.

[14] R. R. Phelps, Lectures on Maximal Monotone Operators, Extracta Mathematicae 12 (1997), 193-230.

[15] R. T. Rockafellar, Extension of Fenchel's duality theorem for convex functions, Duke Math. J. 33 (1966), 81-89.

[16] R. T. Rockafellar, On the maximal monotonicity of subdifferential mappings, Pac. J. Math 33 (1970), 209-216.

[17] S. Simons, Subtangents with controlled slope, Nonlinear Analysis 22 (1994), $1373-1389$.

[18] — , The range of a monotone operator, J. Math. Anal. Appl. 199 (1996), 176-201.

[19] —, Minimax and monotonicity, Lecture Notes in Mathematics 1693 (1998), Springer-Verlag.

[20] —- From Hahn-Banach to monotonicity, Lecture Notes in Mathematics, 1693, second edition, (2008), Springer-Verlag.

[21] — Banach SSD spaces and classes of monotone sets, J. of Convex Anal., 18 (2011), 227-258.

[22] —, A "Density" and maximal monotonicity, arxiv.org/abs/1407.1100v1.

[23] — - "Densities" and maximal monotonicity, J. of Convex Anal., 23 (2016), $1017-1050$.

[24] S. Simons and C. Zălinescu, Fenchel duality, Fitzpatrick functions and maximal monotonicity, J. of Nonlinear and Convex Anal., 6 (2005), 1-22.

[25] S. Simons and X. Wang, Ubiquitous subdifferentials, $r_{L}$-density and maximal monotonicity, Set-Valued Var. Anal. 23 (2014), 631-642. DOI: 10.1007/s11228-015-0326-7.

[26] A. and M. E. Verona, Remarks on subgradients and $\varepsilon$-subgradients, SetValued Analysis 1(1993), 261-272.

[27] M. D. Voisei and C. Zălinescu, Strongly-representable operators, J. of Convex Anal., 16 (2009), 1011-1033. 